Aus der Klinik für Kinder-und Jugendmedizin

(Univ. Prof. Dr. med. Jutta Gärtner)

der Medizinischen Fakultät der Universität Göttingen

\title{
Array-CGH bei Kindern mit Entwicklungsstörung oder geistiger Behinderung: \\ Bei welcher klinischen Konstellation finden sich gehäuft klinisch relevante Chromosomenaberrationen?
}

\author{
Inaugural-Dissertation \\ zur Erlangung des Doktorgrades \\ der \\ Medizinischen Fakultät der \\ Georg-August-Universität zu Göttingen
}

Vorgelegt von

Nina Klein

aus Idar-Oberstein

Göttingen 2016 
Dekan:

Referent/in

Ko-Referent/in:

Drittreferent/in:
Prof. Dr. rer. nat. H. K. Kroemer

Apl. Prof. Dr. med. Dr. rer. nat. B. Zirn

Prof. Dr. med. B. Zoll

Prof. Dr. med. M. Schön

Datum der mündlichen Prüfung: 25.01.2017 
Hiermit erkläre ich, die Dissertation mit dem Titel "Array-CGH bei Kindern mit Entwicklungsstörung oder geistiger Behinderung: Bei welcher klinischen Konstellation finden sich gehäuft klinisch relevante Chromosomenaberrationen?" eigenständig angefertigt und keine anderen als die von mir angegebenen Quellen und Hilfsmittel verwendet zu haben.

Göttingen, den .............. 


\section{Inhaltsverzeichnis}

\section{Abkürzungsverzeichnis}

1. Einleitung .. .1

1.1 Intelligenzminderung: Definition, Klassifikation und Prävalenz...........................1

1.2 Ursachen von Entwicklungsstörungen und Intelligenzminderung ................... 2

1.3 Copy number variations .............................................................................. 4

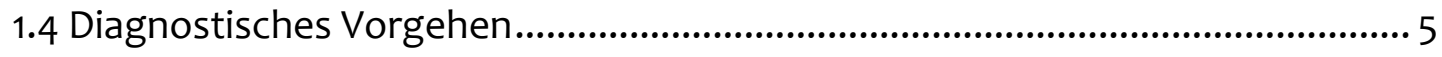

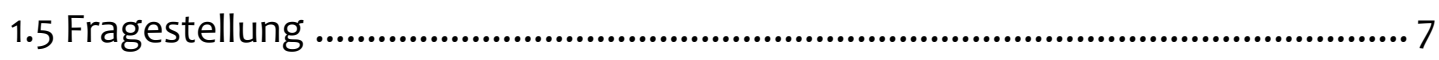

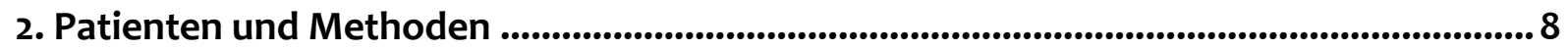

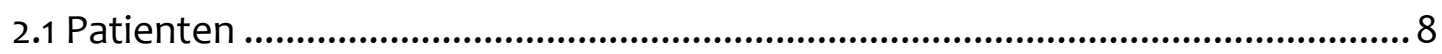

2.2 Auswertung der klinischen Daten ............................................................ 8

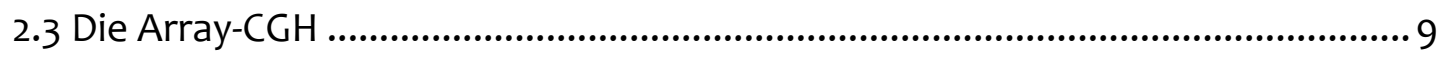

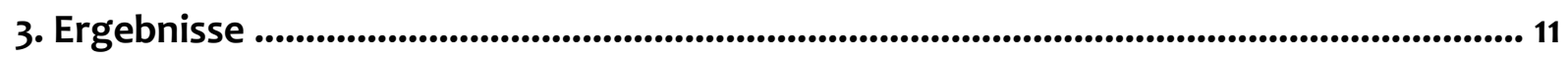

3.1 Genetische Daten ............................................................................................ 11

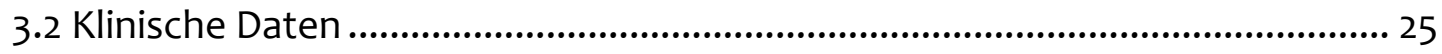

4. Diskussion ................................................................................................................................... 30

5. Ausblick .............................................................................................................................................................. 33

6. Zusammenfassung .................................................................................................................................... 34

7. Literaturverzeichnis ..................................................................................................................... 35

Anhang: Publikation der Daten in Clinical Genetics 


\section{Abkürzungsverzeichnis}

Array-CGH:

Array comparative genomic hybridization

BAC:

Bacterial-artificial- chromosome

CMRT:

Craniale Magnetresonanztomographie

CNV:

Copy number variation

DD:

Developmental delay

Del:

Deletion

DNA:

Desoxyribonukleinsäure

Dup:

Duplikation

EDTA:

Ethylendiamintetraessigsäure

ICD-10: International Classification of Diseases, Version 10

ID: Intellectual disability

IQ: $\quad$ Intelligenzquotient

Kb: $\quad$ Kilobasen

LCR: $\quad$ Low copy repeats

LOH: $\quad$ Loss of heterozygosity

Mb: Megabasen

MLPA: $\quad$ Multiplex ligation-dependent probe amplification

NGS: $\quad$ Next generation sequencing

PCR: $\quad$ Polymerase chain reaction

qPCR: $\quad$ Quantitative polymerase chain reaction

SNP:

Single nucleotide polymorphism 
SPZ:

SSW:

UPD:

XLID:

XLMR:
Sozialpädiatrisches Zentrum

Schwangerschaftswoche

Uniparentale Disomie

X-linked intellectual disability

$X$-linked mental retardation 


\section{Einleitung}

Weltweit sind 1-3\% der Bevölkerung von einer Intelligenzminderung (engl. intellectual disability (ID)) betroffen. Auf Grund der eingeschränkten therapeutischen Möglichkeiten und der lebenslangen Beeinträchtigung der Betroffenen stellt die ID ein bedeutsames Gesundheitsproblem dar (Leonard und Wen 2002). 8 \% der gesamten Gesundheitskosten werden durch Intelligenzminderung verursacht (Ropers und Hamel 2005). Die Ursachen der Erkrankungen sind in bis zu 60 \% aller Fälle unbekannt (Rauch et al. 2006).

\subsection{Intelligenzminderung: Definition, Klassifikation und Prävalenz}

Intelligenzminderung, früher auch als mentale Retardierung bezeichnet (Salvador-Carulla und Bertelli 2008), wird als Zustand von verzögerter oder unvollständiger Entwicklung der geistigen Fähigkeiten definiert (Deutsches Institut für Medizinische Dokumentation und Information 2012). Gemäß der Definition der American Psychiatric Association müssen drei Kriterien für die Diagnose erfüllt sein (American Psychiatric Association 2000):

- $\quad$ ein Intelligenzquotient (IQ) von < 70;

- Manifestation der Symptomatik in der Kindheit, dies entspricht 18 Jahren oder jünger;

- Einschränkungen der Fähigkeiten des täglichen Lebens, wie beispielsweise Kommunikation oder Selbstversorgung.

Die Intelligenzminderung lässt sich nach der International Classification of Diseases, Version 10 (ICD-10) mit Hilfe des Intelligenzquotienten in verschiedene Schweregrade einteilen. Man unterscheidet milde Formen mit einem IQ von 50 - 69, mittelgradige Ausprägungen (IQ 35 - 49), schwere Intelligenzminderung bei Werten von 20 - 34 sowie die schwerste Intelligenzminderung mit einem IQ kleiner 20 (Leonard und Wen 2002). Der Intelligenzquotient wird durch standardisierte, altersspezifische Tests, wie beispielsweise den Hamburg-Wechsler-Intelligenztest für Kinder, ermittelt (Ropers 2008). Bei Säuglingen und Kleinkindern, die altersbedingt noch nicht einem solchen standardisierten Test unterzogen wurden, aber deutliche Verzögerungen der Entwicklung aufweisen, wird von Entwicklungsstörungen gesprochen. 
Entwicklungsstörung und Intelligenzminderungen können isoliert, d.h. ohne weitere spezifische Symptome, oder im Rahmen von syndromalen Erkrankungen auftreten. Bei Letztgenannten treten neben der Intelligenzminderung Dysmorphien (z.B. Hypertelorismus), neurologische Symptome (z.B. Abduzensparese) oder Fehlbildungen (z.B. Herzfehler) auf. Daher ist es oft leichter, syndromale Formen zu diagnostizieren und einer bestimmten Ursache zuzuordnen (Basel-Vanagaite 2008).

Milde Formen der Intelligenzminderung treten in den westlichen Industrieländern mit einer Häufigkeit von etwa $2 \%$ auf. Tiefgreifende Formen, also Erkrankungen mit IQ- Werten kleiner 50, gehen mit einer Prävalenz von 0,3-0,5\% einher. ID ist somit eine durchaus häufig auftretende Erkrankung (Rauch 2010). In Entwicklungsländern scheint die Erkrankung noch häufiger aufzutreten (Durkin 2002, Durkin et al. 1998, Durkin et al. 2000, Costeff et al. 1972), was sich durch vorherrschende Mangelversorgung und unzureichende Gesundheitsversorgung erklären lässt. Des Weiteren spielt hierbei auch ein häufigeres Auftreten von Konsanguinität der Eltern eine Rolle (Ropers 2008). Männer sind öfter von ID betroffen als Frauen (1.4-1.6:1). Dies lässt sich unter anderem damit erklären, dass Männer im Rahmen X-chromosomal bedingter Erkrankungen häufiger betroffen sind (Croen et al. 2001, Curry et al. 1997).

\subsection{Ursachen von Entwicklungsstörungen und Intelligenzminderung}

Die Ursachen einer Intelligenzminderung (Abbildung 1) sind sehr vielfältig. Nur in circa $50 \%$ aller Fälle von tiefgreifender bis mittelgradiger ID lässt sich eine definitive Ursache ermitteln, bei leichten Fällen ist dies seltener der Fall (Chelly et al. 2006). Man unterscheidet zwischen nicht-genetischen (exogenen) und genetischen Faktoren. Zu den nicht-genetischen bzw. exogenen Ursachen zählen unter anderem pre-, peri- und postnatale Ursachen wie Infektionen, Asphyxie, Frühgeburten oder Geburtskomplikationen (Chelly et al. 2006). Bei den genetischen Ursachen wird zwischen großen chromosomalen

Veränderungen, die mit Hilfe konventioneller Chromosomenanalysen sichtbar sind, submikroskopischen Chromosomenaberratio-nen, die sich auf Grund ihre Größe von < 5 Megabasen ( $\mathrm{Mb}$ ) nicht mit klassischen Untersuchungstechniken darstellen lassen, monogenen Störungen, Fehlregulationen imprinteter Gene sowie multifak- 
toriellen Störungen, einer Mischung aus genetischen Faktoren und umweltbedingten Noxen, unterschieden (Basel-Vanagaite 2008, Rauch 2010). In etwa 40 \% der Fälle lässt sich eine eindeutige genetische Ursache der ID ermitteln. Dies bedeutet im Rückschluss, dass in etwa $60 \%$ der Fälle keine eindeutige Ursache gefunden werden kann (Rauch et al. 2006).

Durch den Einsatz neuer Untersuchungsmethoden wird sich die Anzahl dieser Fälle jedoch in Zukunft verringern. Der Nachweis von Punktmutation, die mittels Array-CGH nicht nachweisbar sind, wird beispielsweise durch die Verwendung von Hochdurchsatzverfahren erheblich erleichtert. (Deutsche Gesellschaft für Kinder- und Jugendpsychiatrie, Psychosomatik und Psychotherapie 2014).

In der konventionellen Chromosomenanalyse sichtbare chromosomale Störungen liegen in etwa 15 \% aller Fälle vor (Ropers 2008). Dabei ist das Down-Syndrom, die Trisomie 21, die häufigste Einzelursache für ID mit einer Häufigkeit von circa 9 \% (Collins et al. 2008, Nazer und Cifuentes 2006, Rauch 2010). Mikroaberrationen sind bei 15 \% der Patienten als Ursache für die mentale Retardierung anzusehen. Monogene Ursachen gehen mit einer Häufigkeit von etwa 9 \% einher. Dieser Anteil ist jedoch wahrscheinlich höher (Neveling und Hoischen 2012). Aktuell sind über 400 Genmutationen bekannt, die zu Intelligenzminderung führen können. Es ist allerdings zu erwarten, dass sich diese Anzahl deutlich vervielfachen wird (Van Bokhoven 2011). Seit der Verwendung von Exomsequenzierungen konnten vermehrt De-novo-Mutationen detektiert werden. (Neveling und Hoischen 2012). Diese spielen eine bedeutende ursächliche Rolle in der Entstehung von Intelligenzminderung und Entwicklungsverzögerung. (Vissers et al. 2010). Von besonderem Interesse ist hierbei das X-Chromosom, da mittlerweile bereits über 80 Gendefekte gefunden wurden, die eine $X$-linked intellectual disability (XLID), zuvor als X-linked mental retardation (XLMR) bezeichnet, verursachen (Rauch 2010, Ropers 2008). 


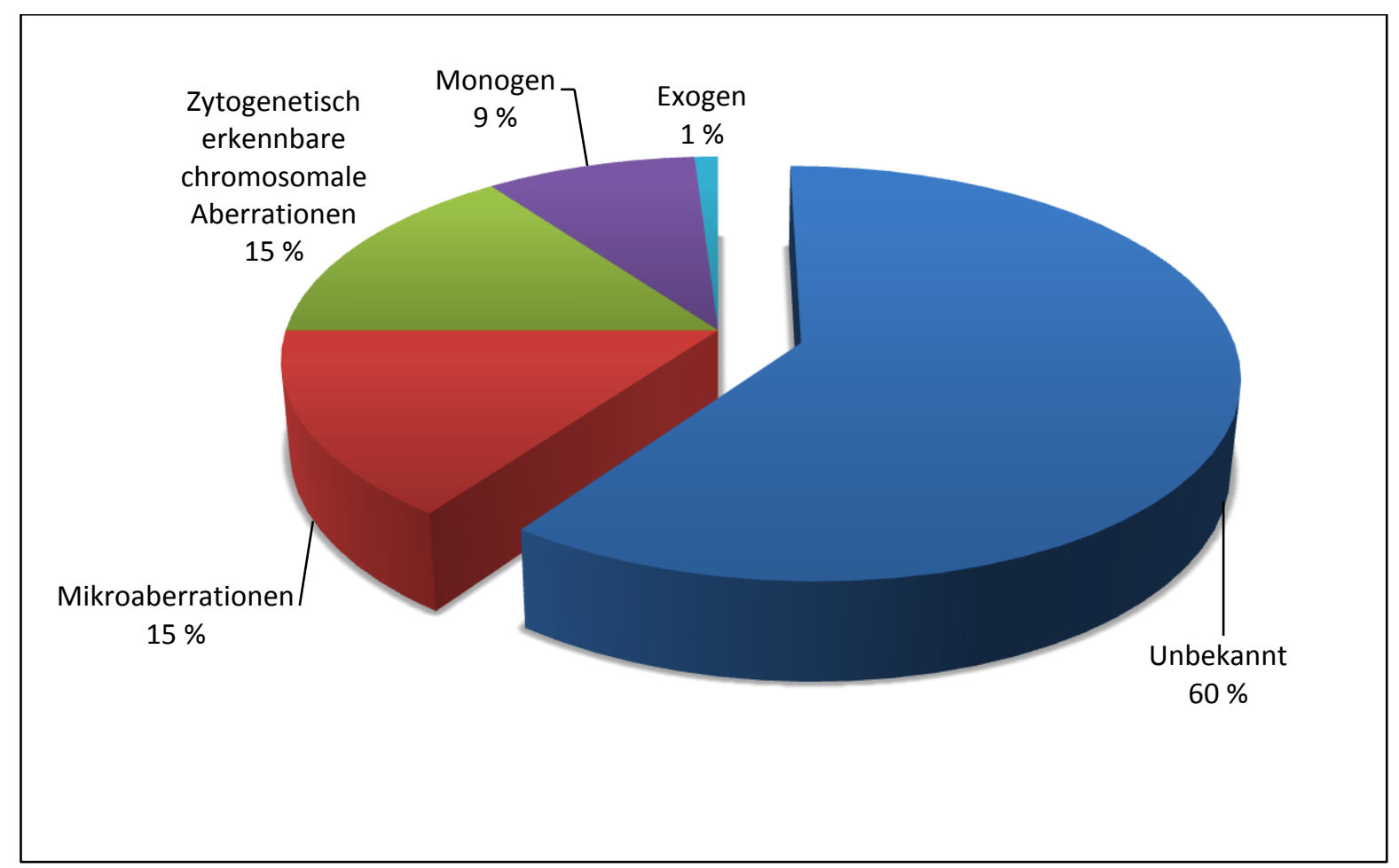

Abbildung 1: Prozentuale Häufigkeiten verschiedener Ursachen von Intelligenzminderung. (Abgewandelt nach Rauch et al. 2006, S. 2071)

\subsection{Copy number variations}

Kopienzahlveränderungen (engl. copy number variations, CNVs) umfassen Duplikationen (Chromosomenstückverdopplungen) und Deletionen (Chromosomenstückverluste) eines Abschnitts Desoxyribonukleinsäure (DNA) in Bezug auf eine Vergleichsprobe des menschlichen Genoms. CNVs können von unterschiedlicher Größe sein und reichen von einigen Kilobasen (Kb) bis hin zu mehreren Megabasen oder umfassen sogar ganze Chromosomen (Mefford et al. 2012). Es wurde nachgewiesen, dass CNVs an unterschiedlichen Positionen des Genoms unterschiedlich häufig vorkommen. Prädisponierend für das Auftreten von c ist die Flankierung durch low copy repeats (LCRs), die Deletionen (Del) und Duplikationen (Dup) durch mitotische Fehlanlagerung der Chromosomen auslösen können. Einige CNVs haben keinen Krankheitswert (Lafrate et al. 2004, Itsara et al. 2009, Locke et al. 2006, Redon et al. 2006, Sebat et al. 2004). Sogar in genkodierenden Regionen sind CNVs beschrieben, die mit keiner klinischen Manifestation einhergehen. Diese Polymorphismen treten mit einer Frequenz von $>1 \%$ in der Bevölkerung auf (Gillesen-Kaesbach und Siebert 2012). Viele CNVs sind jedoch verantwortlich für Intelligenzminderungen, 
Entwicklungsverzögerungen oder andere Erkrankungen (Mefford et al. 2012). CNVs lassen sich daher in verschiedene Kategorien unterteilen. Eine Kopienzahlveränderung, die ein bereits bekanntes Mikrodeletions- oder Mikroduplikationssyndrom verursacht, bereits in der Literatur beschrieben ist oder die sich bei mindestens zwei Patienten mit äquivalentem Phänotyp in Datenbanken finden lässt, bezeichnet man als pathogen. Als nicht pathogen hingegen bezeichnet man CNVs, die als häufige Normalvariante in der Bevölkerung auftreten. Es gibt aber auch detektierte Veränderungen unklarer Signifikanz. Diese versucht man dann in eher pathogen und eher nicht pathogen zu differenzieren. Dabei kann die molekulare Karyotypisierung der Eltern weiterhelfen. Eine Kopienzahlveränderung, die von einem gesunden Elternteil vererbt wurde, gilt beispielsweise als eher nicht pathogen. Allerdings sollte dabei das Vorliegen von verminderter Penetranz oder variabler Expressivität nicht außer Acht gelassen werden. Die klinische Interpretation von CNVs unklarer Signifikanz gestaltet sich daher häufig problematisch (Spengler et al. 2013). Datenbanken zum Abgleich der detektierten CNVs sind dabei ein wichtiges Hilfsmittel (Oneda und Rauch 2012).

\subsection{Diagnostisches Vorgehen}

Bei allen Kindern mit Verdacht auf Entwicklungsstörungen oder Intelligenzminderung sollten - wie in Abbildung 2 ersichtlich - zunächst die Sicherung der Diagnose durch standardisierte Intelligenz- und Entwicklungstest sowie eine umfassende klinische und neurologische Untersuchung erfolgen (Miclea et al. 2015). Dabei wird z.B. nach Dysmorphien oder neurologischen Auffälligkeiten gesucht, die hinweisend auf bestimmte Syndrome sein können (Basel-Vanagaite 2008). Das Vorliegen bestimmter Dysmorphien führt gehäuft zur Ursachenklärung (Van Karnebeek et al. 2005). Parameter wie etwa der Kopfumfang, die Körpergröße oder der Muskeltonus können wichtige Hinweise auf eine mögliche Ursache liefern. Kleinwuchs kann beispielsweise in Kombination mit spezifischen fazialen Auffälligkeiten und einer Mikrozephalie hinweisend auf eine fetale Alkoholembryopathie sein, Hochwuchs kommt vermehrt beim Fragilen X- Syndrom vor (Miclea et al. 2015). Gleichzeitig sollten sowohl eine umfassende Eigen- als auch Familienanamnese erhoben werden, um mögliche weitere Fälle von Entwicklungsverzögerungen und Intelligenzminderung in der Familie zu erfassen und somit die Ursachensuche über das Vorliegen be- 
stimmter Erbgänge zu erleichtern (Basel-Vanagaite 2008).

Ergibt sich schließlich der Verdacht auf das Vorliegen eines monogenetischen Syndroms, dann kann eine gezielte genetische Untersuchung auf eben diese Erkrankung erfolgen. Lassen der vorliegende Phänotyp sowie der erhobene Untersuchungsbefund keine Verdachtsdiagnose zu, kommen ungezielte genetische Screeningverfahren - wie z.B, der Array-CGH - zum Einsatz. Auf diese Weise versucht man, eine genetische Mutation oder Chromosomenstörung zu finden, die in einen kausalen Zusammenhang zur vorliegenden Entwicklungsstörung bzw. Intelligenzminderung gebracht werden kann. Diese Vorgehensweise wird als reverse Genetik bezeichnet (Zirn 2012, Willemsen und Kleefstra 2014).

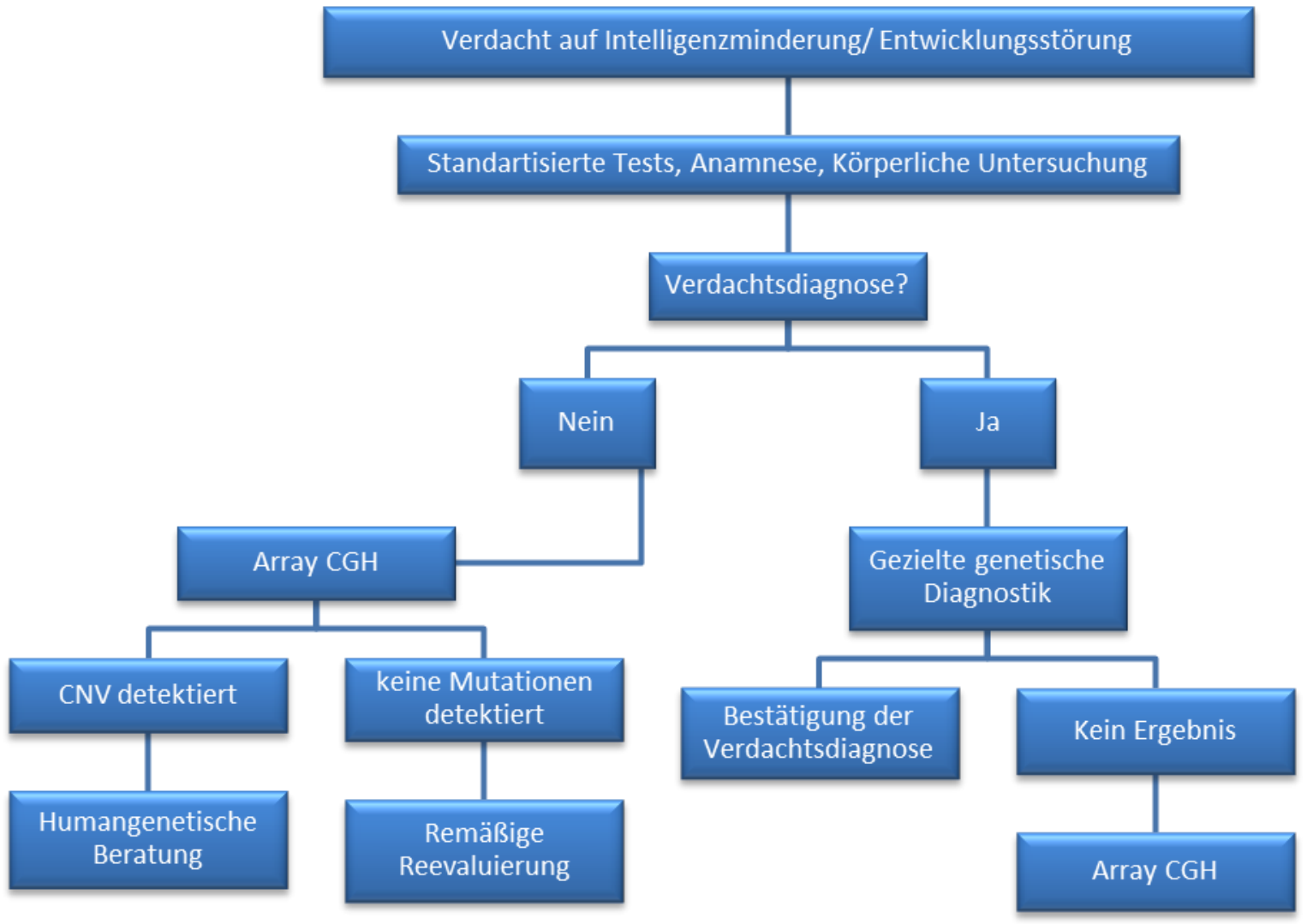

Abbildung 2: Diagnostisches Vorgehen bei Patienten mit dem Verdacht auf ID (Willemsen und Kleefstra 2014, O‘ Byrne et al. 2016, Miclea et al. 2015). 
Als genetische Screening-Verfahren stehen die konventionelle Chromosomenanalyse sowie seit einigen Jahren die array comparative genomic hybridization (Array-CGH) zur Verfügung. Seit 2010 wird die Array-CGH als primäres genetisches Screening-Verfahren zur Abklärung von Entwicklungsstörungen und Intelligenzminderung empfohlen (Miller et al. 2010).

\subsection{Fragestellung}

Die vorliegende Arbeit verfolgt zwei Ziele:

1. das Spektrum phänotypischer Auffälligkeiten, die mit klinischen relevanten Kopienzahlvarianten in Verbindung stehen, zu erweitern;

2. klinische Kriterien zu definieren, die in Zukunft dabei helfen können, Patienten mit klinisch relevanten CNVs von denen ohne pathologische Aberrationen zu unterscheiden. 


\section{Patienten und Methoden}

\subsection{Patienten}

In einem Zeitraum von dreieinhalb Jahren (01.01.2007 bis 01.03.2011) wurden 342 Kinder, die mit Entwicklungsstörungen und Intelligenzminderungen im Sozialpädiatrischen Zentrum (SPZ) der Universitätsmedizin Göttingen zur Ursachenabklärung vorgestellt wurden, mittels Array-CGH untersucht. Die Array-CGH-Untersuchungen wurden im hiesigen Institut für Humangenetik in Kooperation mit Herrn Dr. med. Moneef Shoukier durchgeführt. Im Rahmen einer retrospektiven Studie wurden sowohl die genetischen Untersuchungsergebnisse als auch alle verfügbaren klinischen Daten der Kinder zusammengestellt. Es handelte sich um 227 Jungen und 115 Mädchen. Die Eltern wurden ausführlich vor der Untersuchung aufgeklärt und haben schriftlich in die Untersuchungen eingewilligt.

\subsection{Auswertung der klinischen Daten}

Alle klinischen Daten und die Körpermaße bei Geburt sowie im Verlauf wurden mit Hilfe eines standardisierten Protokolls (Excel-Tabelle) erfasst und ausgewertet. Bezüglich der klinischen Daten wurden folgende Parameter erfasst: Geschlecht, Alter, Fehlbildungen, Epilepsie, Herzfehler, Schwangerschaftsdauer, APGAR-Werte, primäre und sekundäre Mikrozephalie, primäre und sekundäre Makrozephalie, primärer und sekundärer Minderund Hochwuchs, Über- und Untergewicht bei Geburt und im Verlauf, Knochenalter, erstgradige Verwandte mit Entwicklungs-störung oder Intelligenzminderung, Befunde cranialer Magnetresonanztomographie (CMRT), stattgefundene Chromosomenanalysen und genetische Untersuchungen auf spezielle Syndrome.

Alle statistischen Analysen wurden mit Hilfe der Software Statistica 9.1 (Stat-Soft Inc., Tulsa, OK) angefertigt. Die klinischen Befunde der Kinder mit copy number variations und Translokationen eindeutiger klinischer Relevanz wurden mit Hilfe des Chi-Quadrat Tests mit denen der Kinder mit unauffälligem Array-CGH-Befund verglichen. War die erwartete Anzahl kleiner 5, wurde der Chi- Quadrat- Test mit Yates- Korrektur angewendet. Des Weiteren wurde die Bonferroni- Methode verwendet, um das Signifikanzniveau zu beurteilen (Shoukier et al. 2013). 


\subsection{Die Array-CGH}

Während der letzten Jahre hat die Etablierung der Array-CGH die Diagnostik von Intelligenzminderungen und Entwicklungsstörungen unklarer Genese revolutioniert und wurde zu einer Untersuchung erster Wahl (Manning und Hudgins 2010, Miller et al. 2010). Mit Hilfe dieses Verfahrens können eine im Vergleich zur konventionellen Karyotypisierung 10ofach größere Auflösung erreicht und somit auch submikroskopische Chromosomenaberrationen aufgedeckt werden (Miller et al. 2010). Während mit den klassischen Methoden nur Veränderungen zwischen 5 - 10 Megabasen erkannt werden, können mit Hilfe der Array-CGH sogar noch Mutationen im Bereich von 100 - 200 Kb erfasst werden (BaselVanagaite 2008).

Zur Durchführung der Array CGH wird genomische DNA des Patienten extrahiert und mit einem Fluoreszenzfarbstoff (gebräuchlich ist Cy3) markiert. Diese gekennzeichnete Probe wird in gleichem Verhältnis mit einer Kontrollprobe, die mit einem anderen Farbstoff (Cy5) markiert ist, auf einen Chip aufgetragen. Auf dem Chip sind außerdem eine definierte Anordnung tausender immobilisierter DNA- Sonden aufgetragen und hybridisiert. Als Sonden eignen sich beispielsweise bacterial artificial chromosome (BAC) - Klone, Oligonukleotide oder polymerase chain reaction (PCR) -Fragmente. Jede dieser Sonden enthält etwa 70 - 180 Kilobasen des menschlichen Genoms. Die Sequenz und Lage dieser DNA- Fragmente im menschlichen Genom ist bekannt. Nach abgeschlossener Hybridisierung wird nicht gebundenes Material durch verschiedene Waschschritte entfernt und der Chip mit einem Laserscanner untersucht. Die Intensität der Fluoreszenz der Farbstoffe wird mit einer speziellen Software ausgewertet: Sind Patientenmaterial und Referenzprobe an einem Spot gleichermaßen vorhanden, erscheint diese Stelle in einer bestimmten Farbe, wie in Abbildung 3 ersichtlich. Herrscht ein Überschuss an Patienten-DNA, im Falle einer Duplikation einer bestimmten Sequenz, so verschiebt sich das Signal in Richtung Patientenfarbstoff und stellt sich z.B. rot dar. Sollte ein Überschuss der Referenzprobe vorhanden sein, wie im Falle einer Deletion beim Patienten, überwiegt das Signal der Kontrollprobe und der Spot erscheint in Grün. Mit Hilfe der speziellen Software ist eine Lokalisation der deletierten bzw. duplizierten Bereiche im Genom möglich (de Ravel et al. 2007, Feuk et al. 2006, Klein und Rost 2012). 


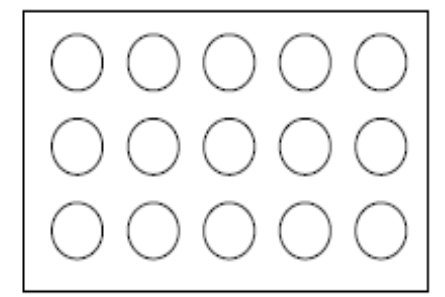

Objektträger (Chip) mit $>100.000$ DNA-Abschnitten

Genomweite Repräsentation

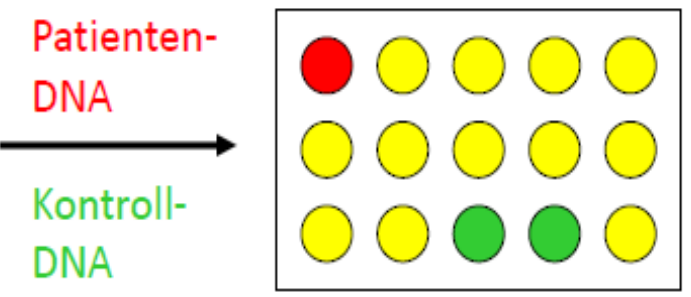

Normales Verhältnis

Zugewinn (Duplikation)

Verlust (Deletion)

Abbildung 3: Schematischer Ablauf einer Array-CGH.

Die genomische DNA wurde aus Ethylendiamintetraessigsäure (EDTA) - Blutproben der Patienten mit Hilfe von Standardtechniken extrahiert. Ein genomweiter copy number Scan erfolgte unter Verwendung des Agilent 244K-Arrays oder des Agilent SurePrint G3 Human-CGH-Microarray Kit 4x 180K (Agilent Technologies, Inc., Santa Clara, CA). Dabei wurden die Anweisungen der Hersteller befolgt. Die Array CGH-Daten wurden mit der Agilent DNA Analytics 4.0 Software ausgewertet. Die Ergebnisse wurden dann mit bekannten copy number variations der Database of Genomic Variants (DGV, http://projects.tcag.ca/variation), der International Standard Cytogenomic Array Consortium Database (https://isca.genetics.emory.edu) sowie der Database of Chromosomal Imbalance and Phenotype in Human using Ensembl Resources (DECIPHER, https://decipher.sanger.ac.uk/application/) verglichen. Pathologische und potentiell pathologische CNVs wurden nochmals überprüft mit Hilfe von multiplex ligation-dependent probe amplification (MLPA, MRC-Holland, Amsterdam, Niederlande), falls das entsprechende Kit verfügbar war. Alternativ wurden die pathologischen Auffälligkeiten mit Hilfe von quantitative polymerase chain reaction $(q P C R)$ überprüft. 


\section{Ergebnisse}

\subsection{Genetische Daten}

Bei 72 von 342 (21,1\%) Kindern mit Entwicklungsstörungen oder Intelligenzminderung wurden Kopiezahlvarianten (CNVs) mittels Array-CGH ermittelt. Davon waren 49 Patienten männlichen Geschlechts, 23 waren weiblich. Es liegen Daten von Kindern und Jugendlichen im Alter von 3 Monaten bis 20 Jahren vor.

Die festgestellten Kopiezahlveränderungen verteilen sich, wie in Abbildung 4 ersichtlich, auf die verschiedenen Chromosomen. Die meisten CNVs finden sich dabei auf dem XChromosom. Dort wurden drei Deletionen und vier Duplikationen detektiert. Auf Chromosom 18 wurden keine Kopiezahlvarianten festgestellt. Die meisten Deletionen zeigen sich auf Chromosom 4 (6 Stück). Die größte Anzahl von Duplikationen wurde auf dem XChromosom gefunden (4 Stück).

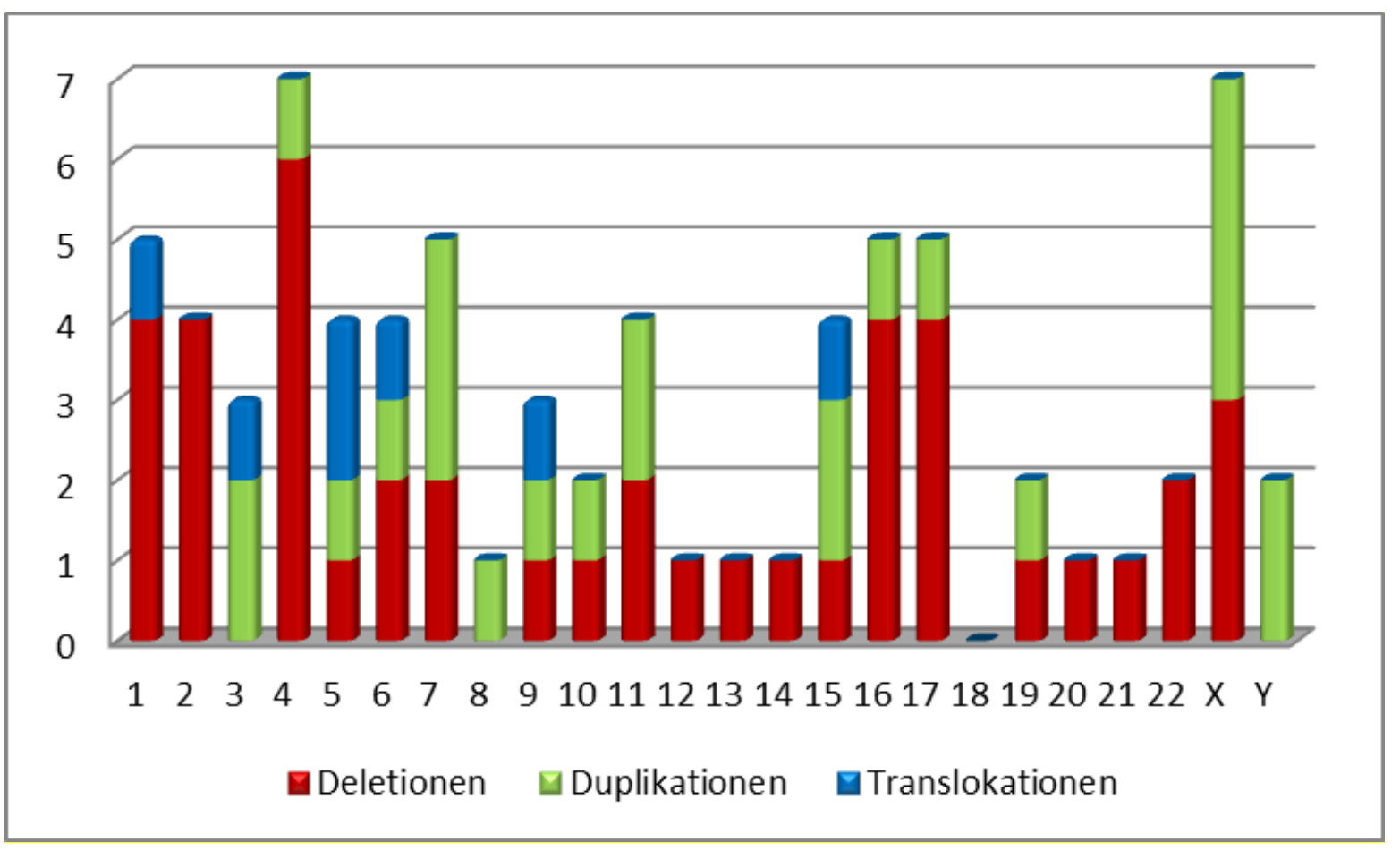

Abbildung 4: Verteilung der CNVs auf den Chromsomen.

Die CNVs lassen sich des Weiteren in verschiedene Gruppen unterteilen (Abbildung 5): Mikrodeletionen und -duplikationen eindeutiger klinischer Relevanz (Gruppe 1) fanden 
sich bei 42 Kindern (58\%). Unbalancierte Translokationen waren bei 3 Kindern (4\%) nachweisbar (Gruppe 2). In Gruppe 3 sind 22 Kinder (31\%) mit Kopiezahlveränderungen unklarer klinischer Bedeutung zusammengefasst. Diese Befunde waren unklar, weil sie entweder bislang nicht in der Literatur beschrieben oder von einem gesunden Elternteil vererbt wurden. Die Befunde der Gruppen 4 und 5 haben keine kausale Bedeutung für die schwere Entwicklungsstörung oder Intelligenzminderung der Kinder. Gruppe 4 enthält 2 Jungen (3 \%) mit Klinefelter-Syndrom (47, XXY-Karyotyp) oder 47, XYY-Karyotyp. In Gruppe 5 sind 3 Kinder ( $4 \%$ ) mit Polymophismen zusammengefasst. Dies sind Kopiezahlveränderungen, die zuvor auch bei Gesunden beschrieben wurden und daher mit großer Wahrscheinlichkeit keine Bedeutung haben. Die klinischen und genetischen Daten der 72 Kinder mit CNVs sind in Tabelle 1a - j zusammengefasst. Insgesamt wurden 44 Deletionen, 25 Duplikationen und 3 Translokationen festgestellt, wie Abbildung 6 zeigt.

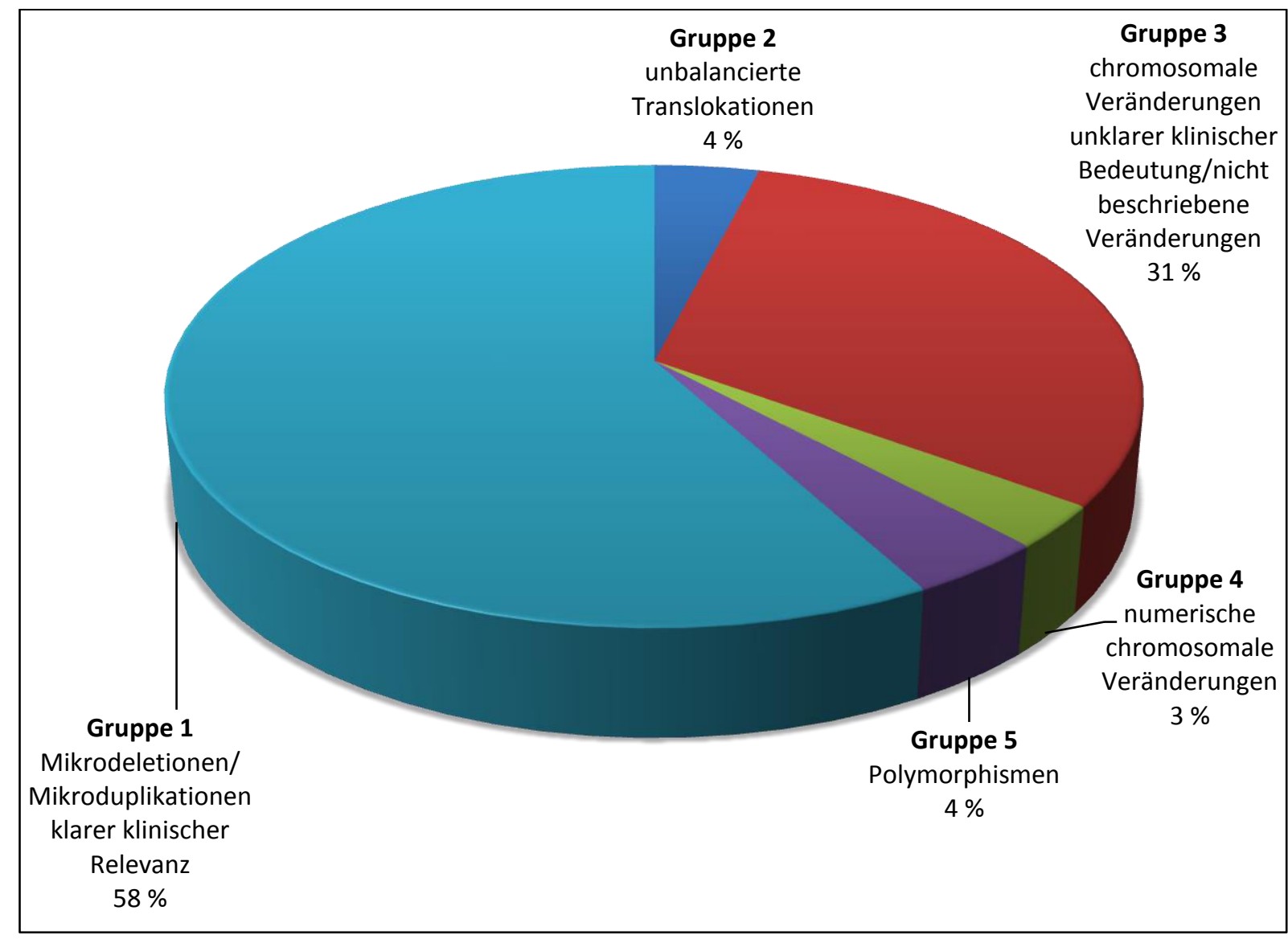

Abbildung 5: Einteilung der 72 Patienten mit auffälligem Array-CGH- Ergebnis in verschiedene Gruppen genetischer Aberrationen. 
Bei strukturellen chromosomalen Veränderungen, die nur ein Chromosomensegment betreffen, treten Deletionen (67\%) häufiger auf als Duplikationen (33\%). Noch häufiger treten Deletionen mit 79\% (33/42) in der Gruppe der bekannten Mikrodeletionssyndrome auf, wohingegen in Gruppe 3, den CNVs unklarer klinischer Relevanz, Duplikationen bei 13 von 22 Patienten und somit in 59\% der Patienten zu finden waren. Der größte Teil der strukturellen Aberrationen war interstitiell, nur in 5\% der Fälle waren die TelomerRegionen beteiligt. Allerdings waren die Telomer-Regionen bei allen der drei unbalancierten Translokationen betroffen. 


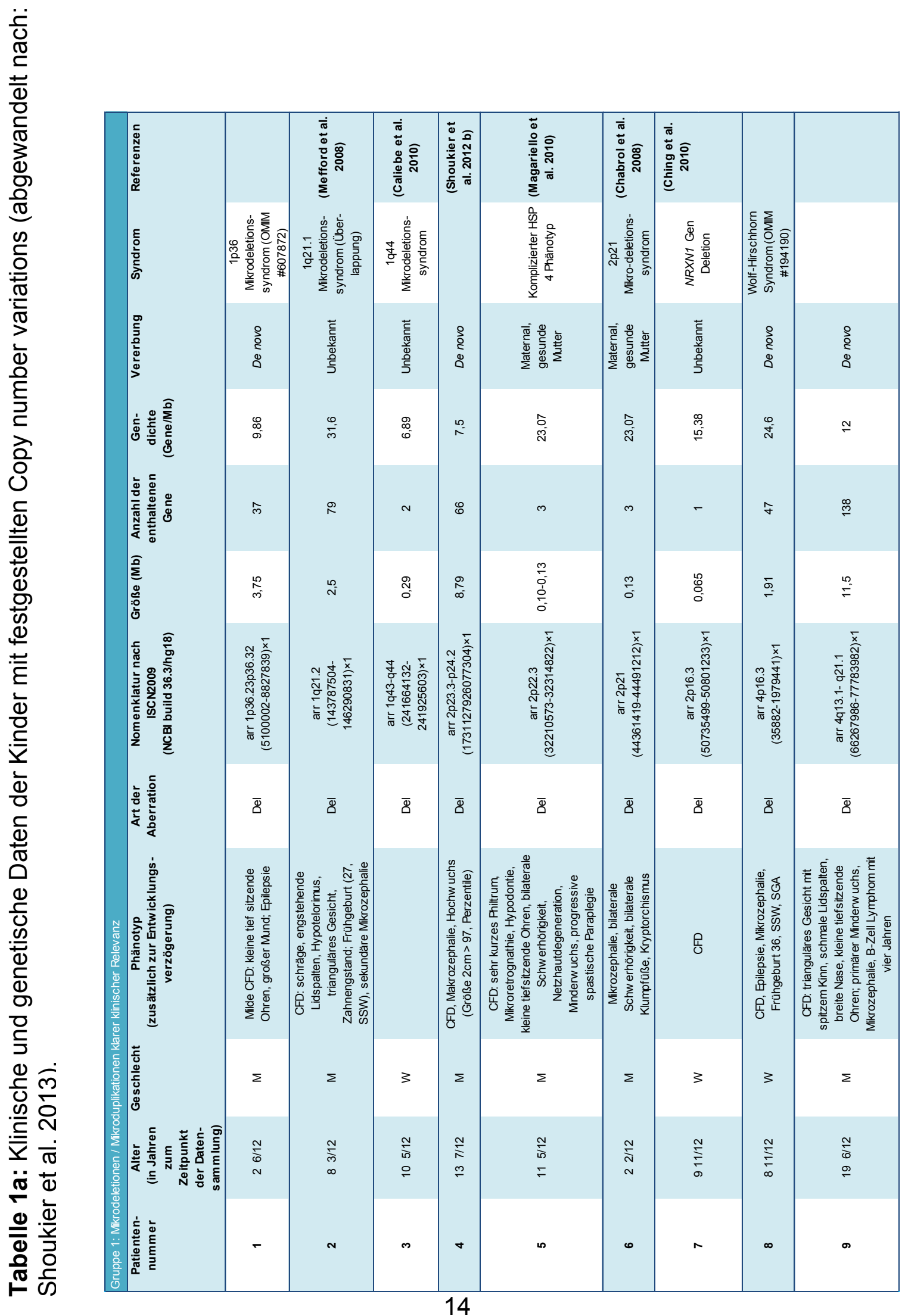




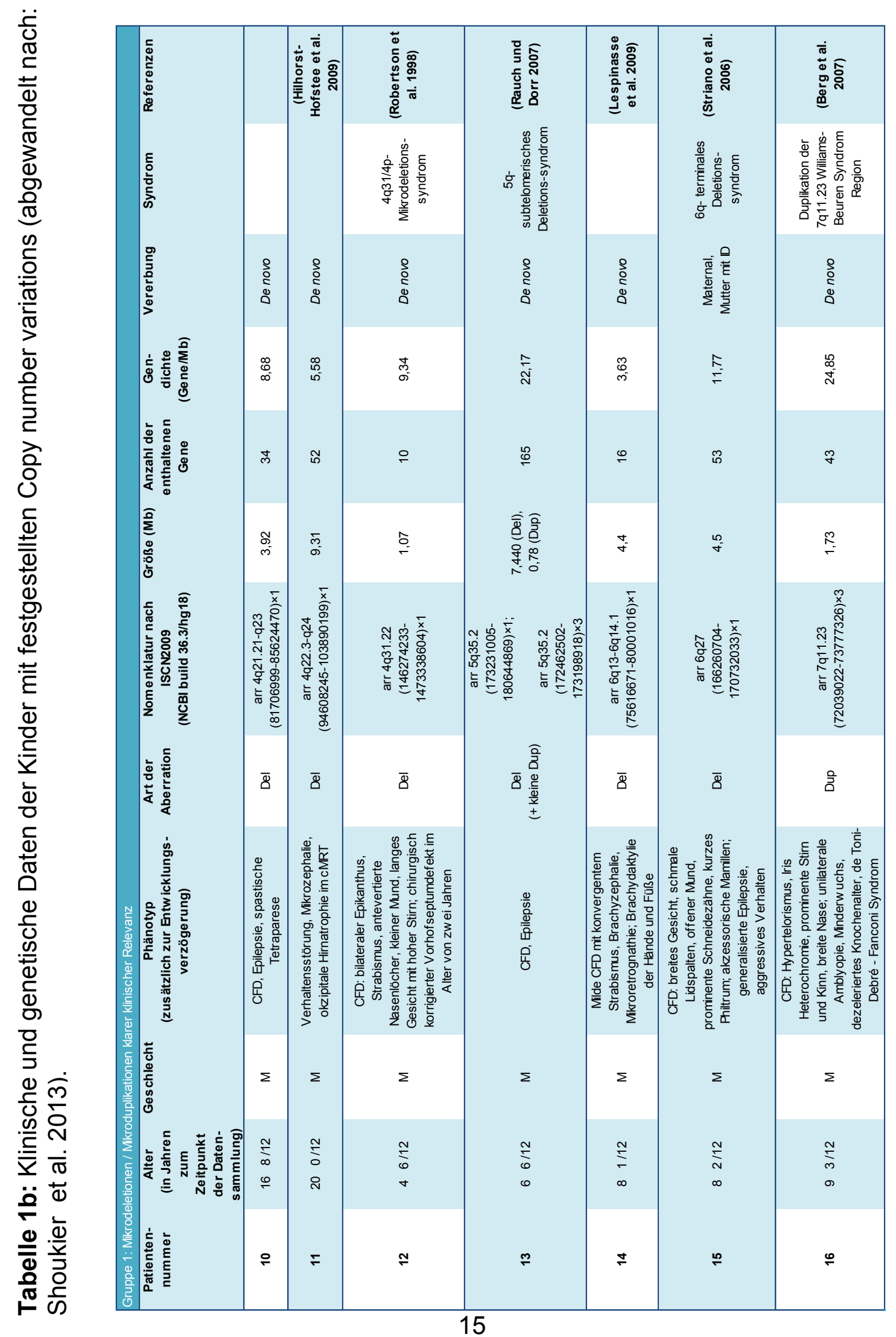




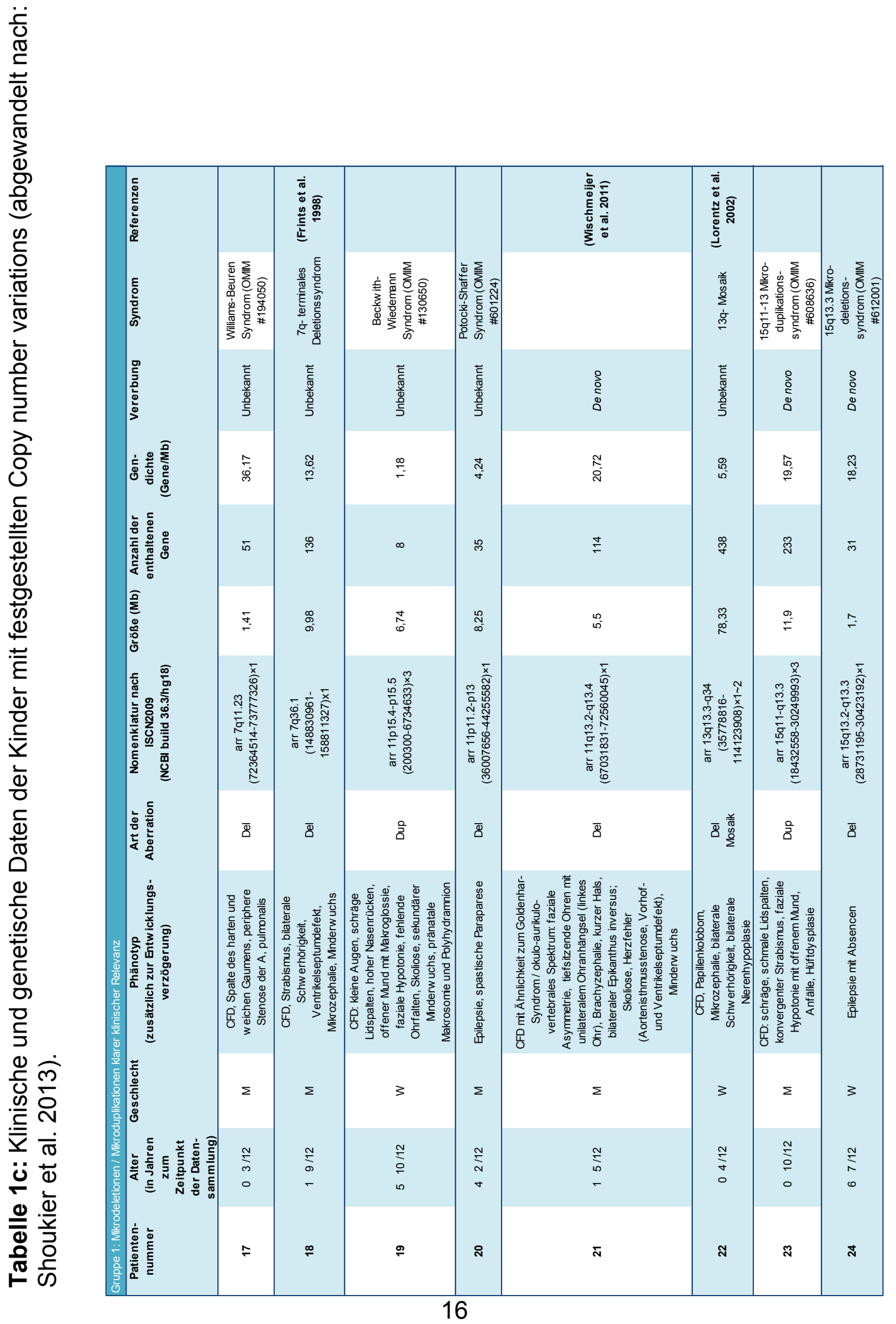




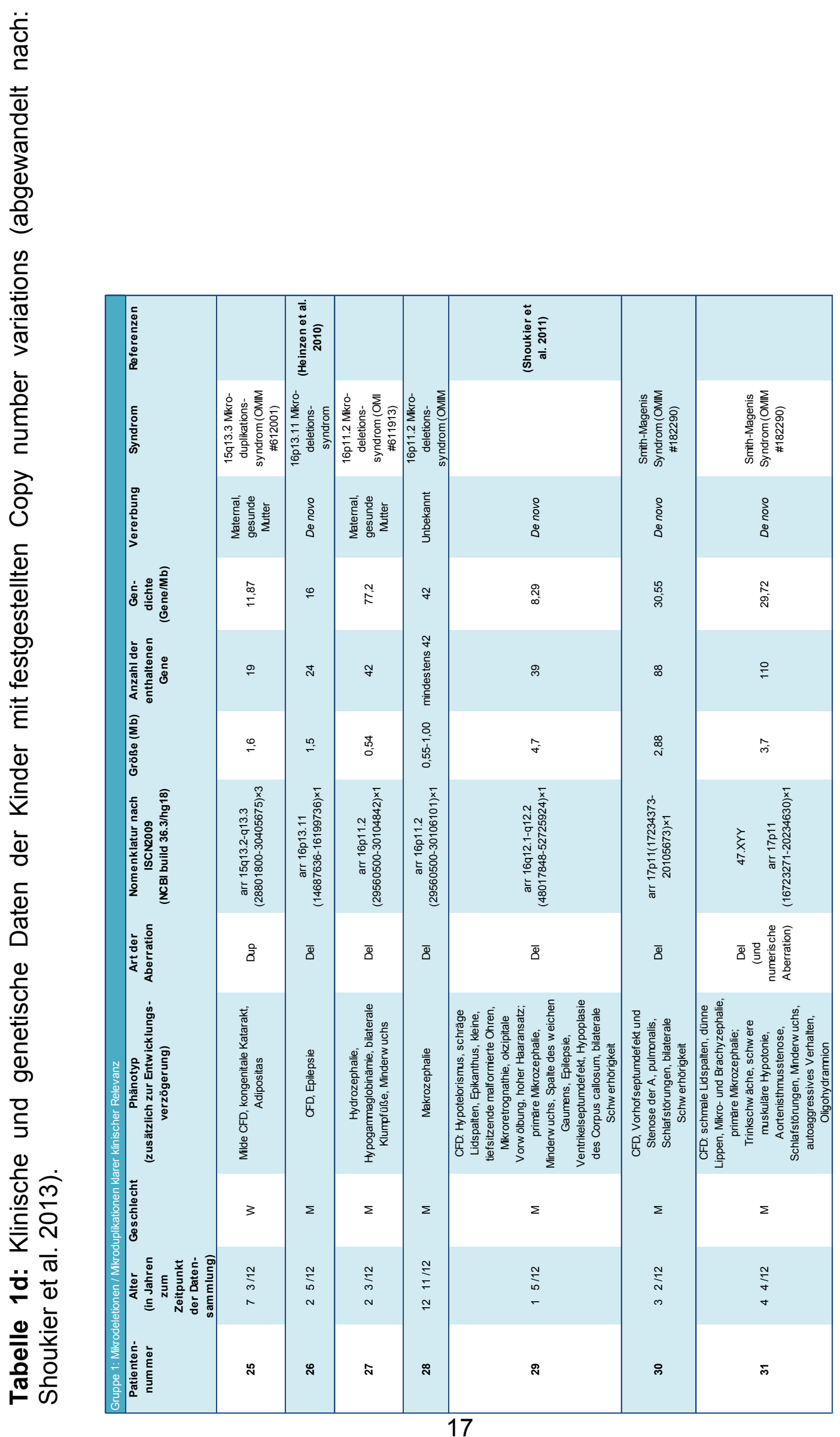




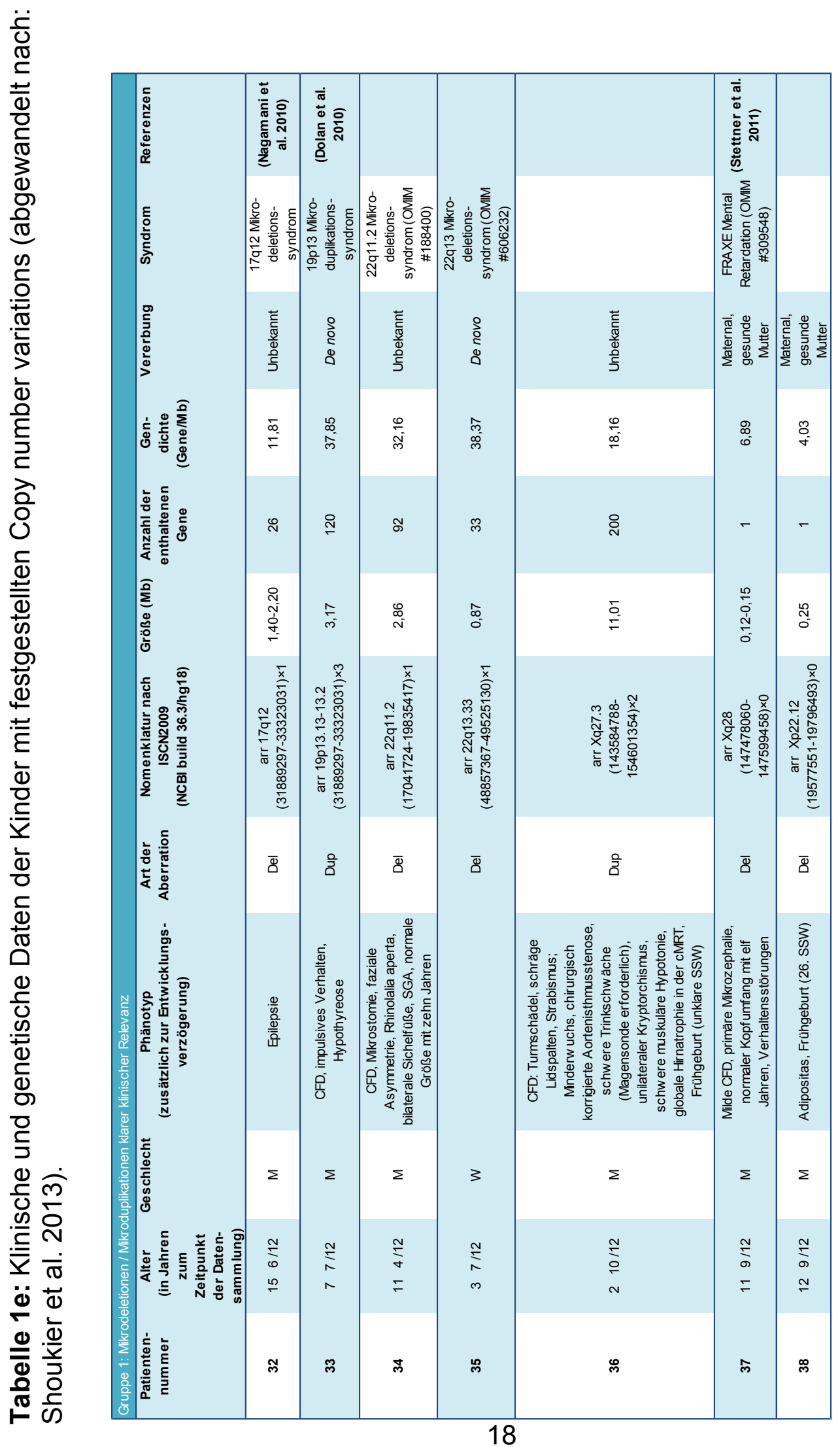




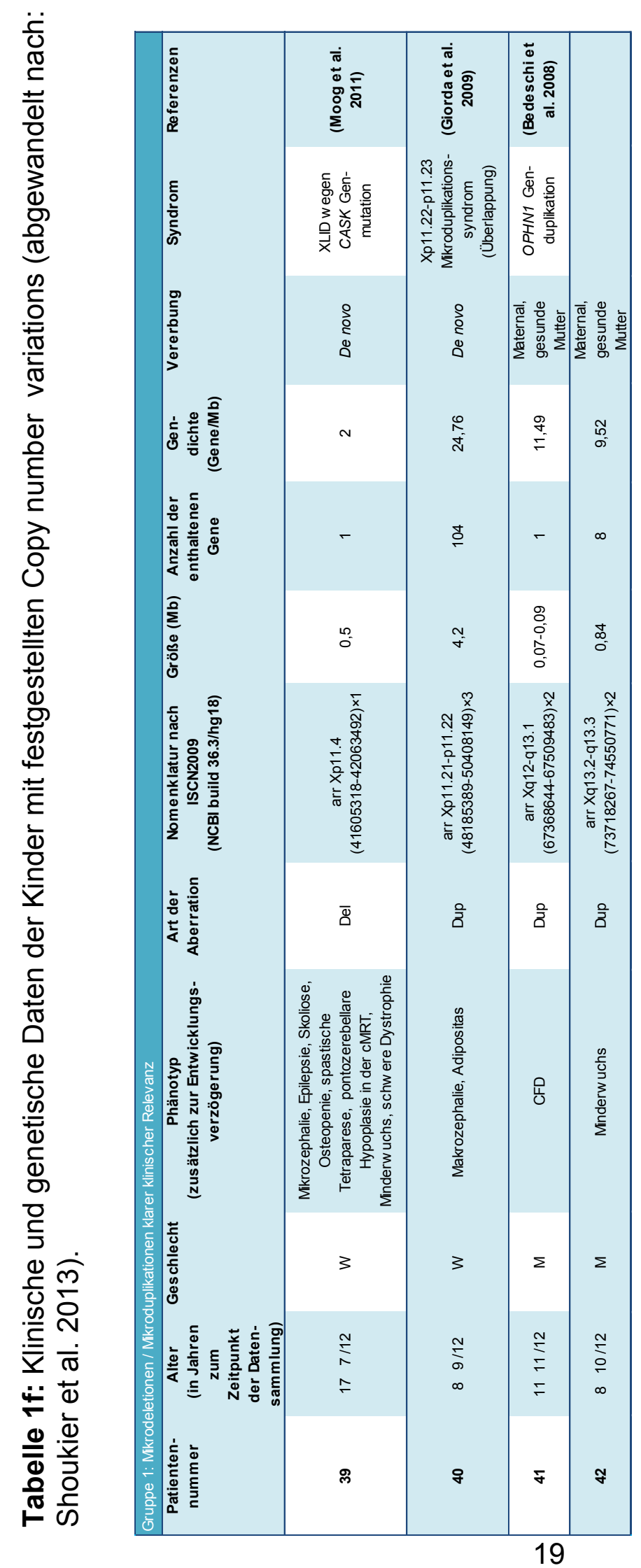




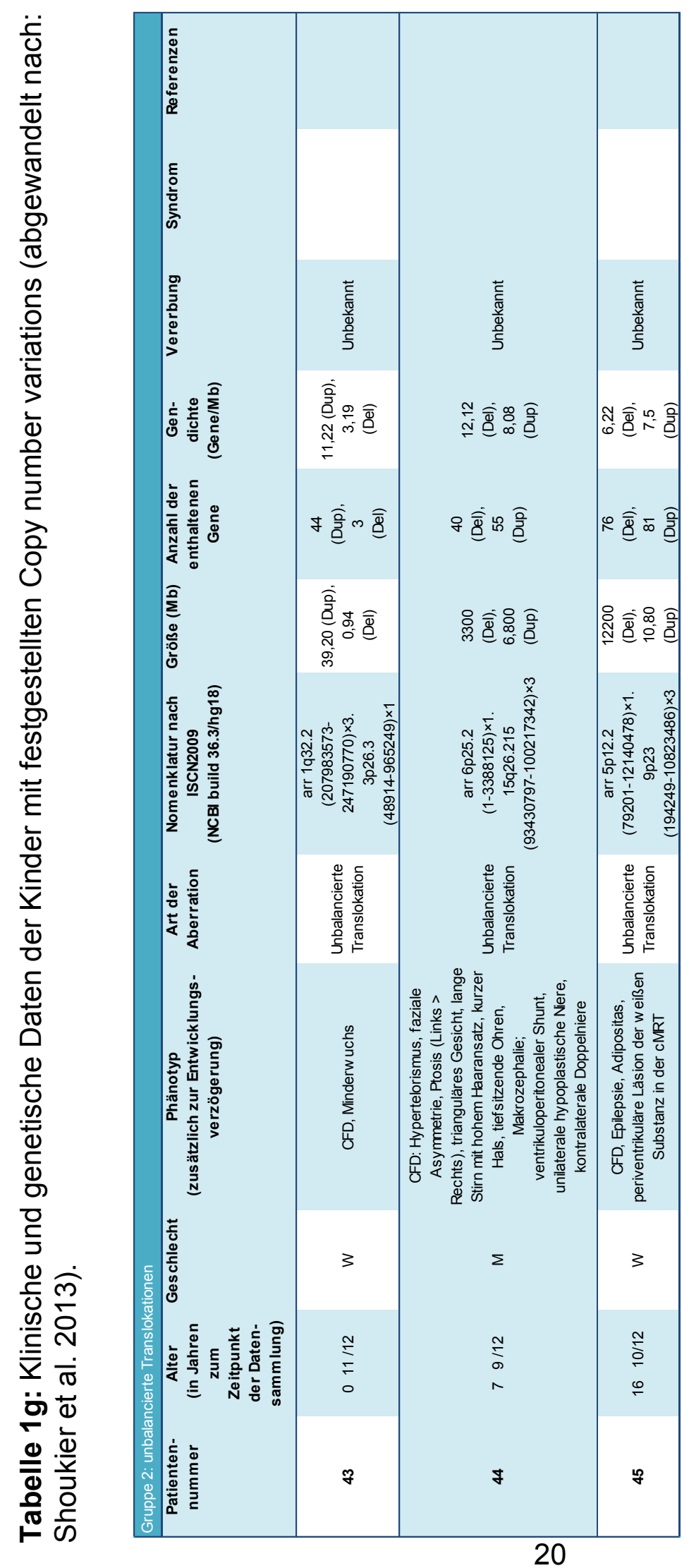




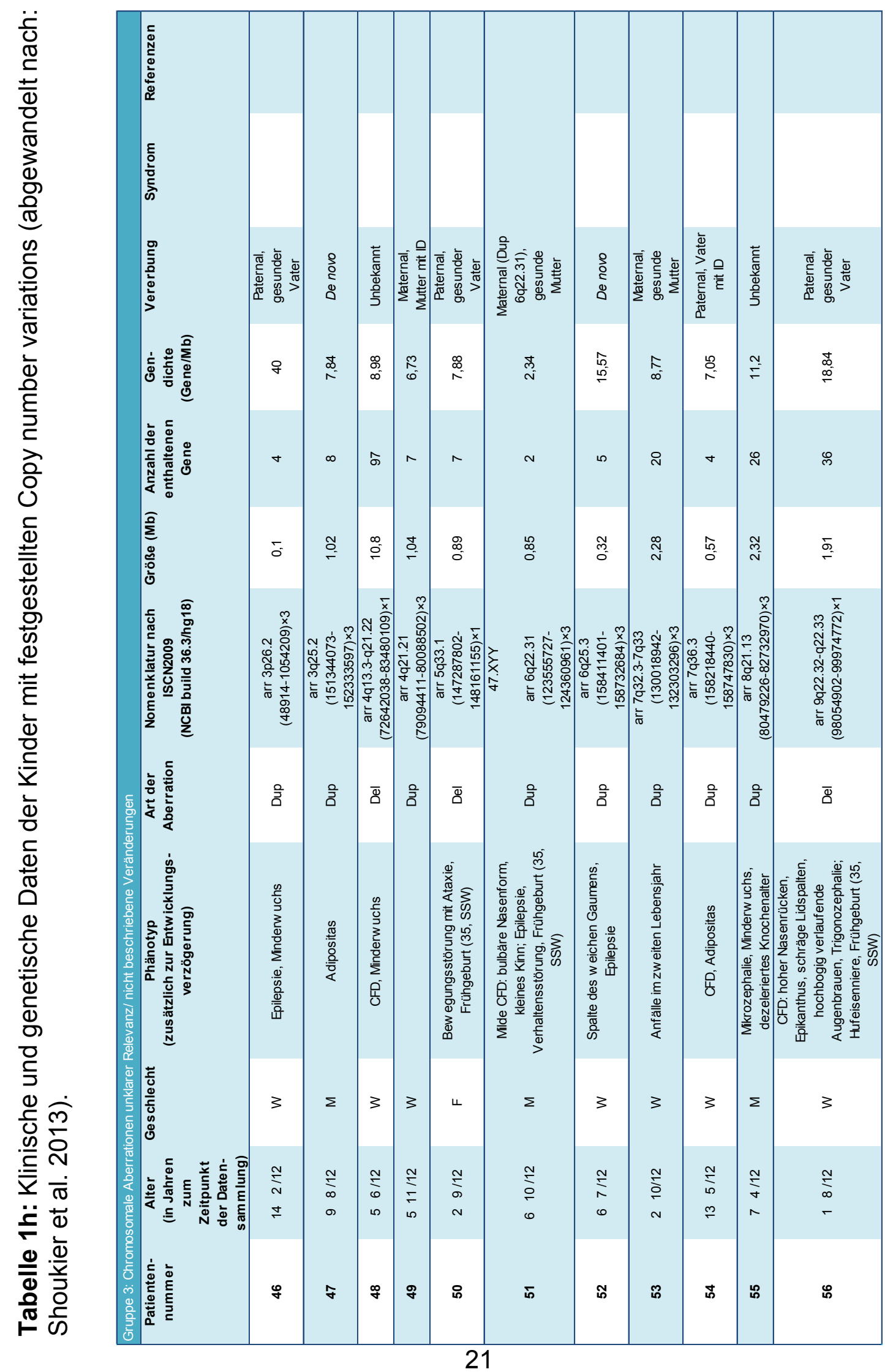




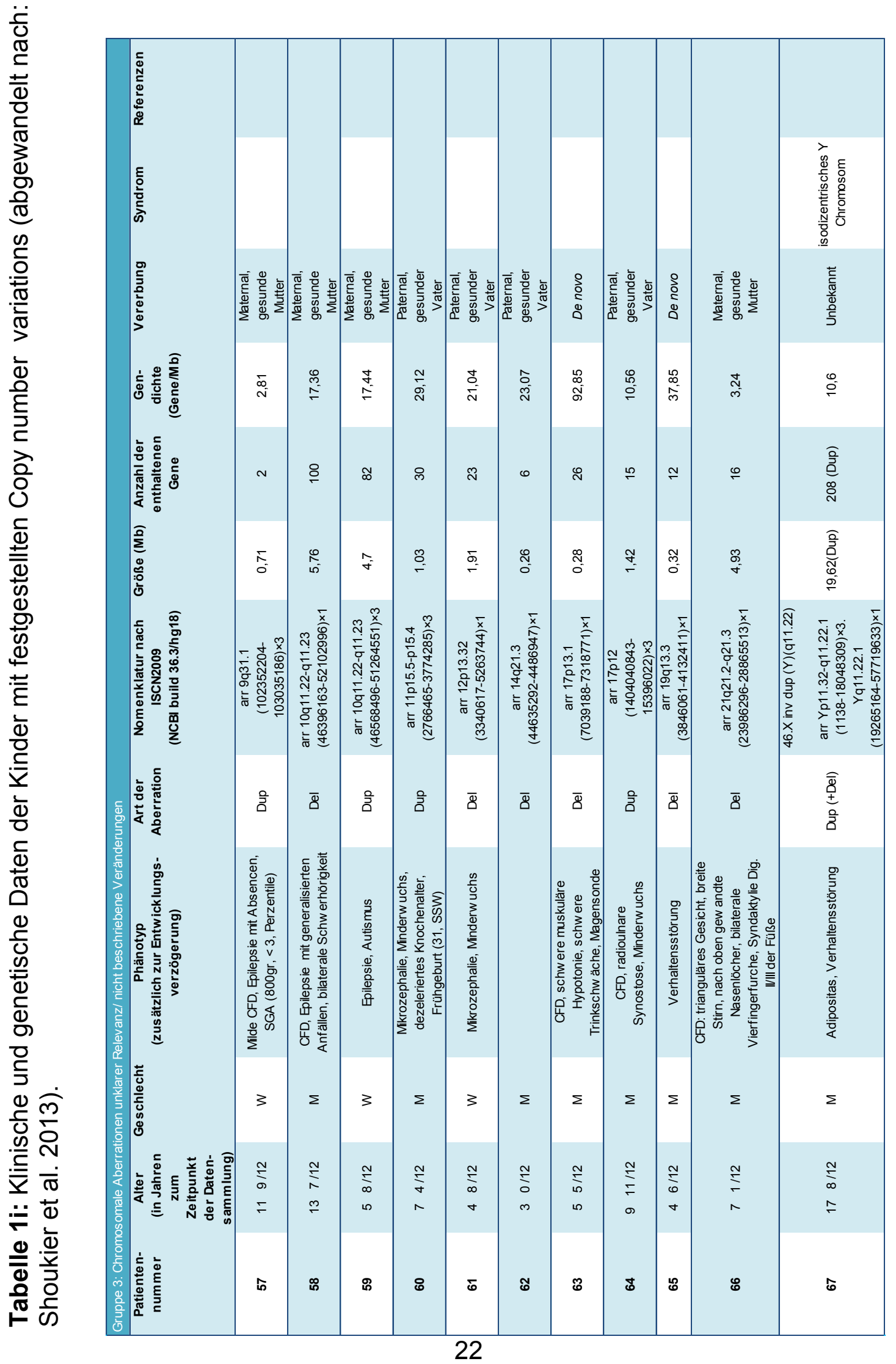



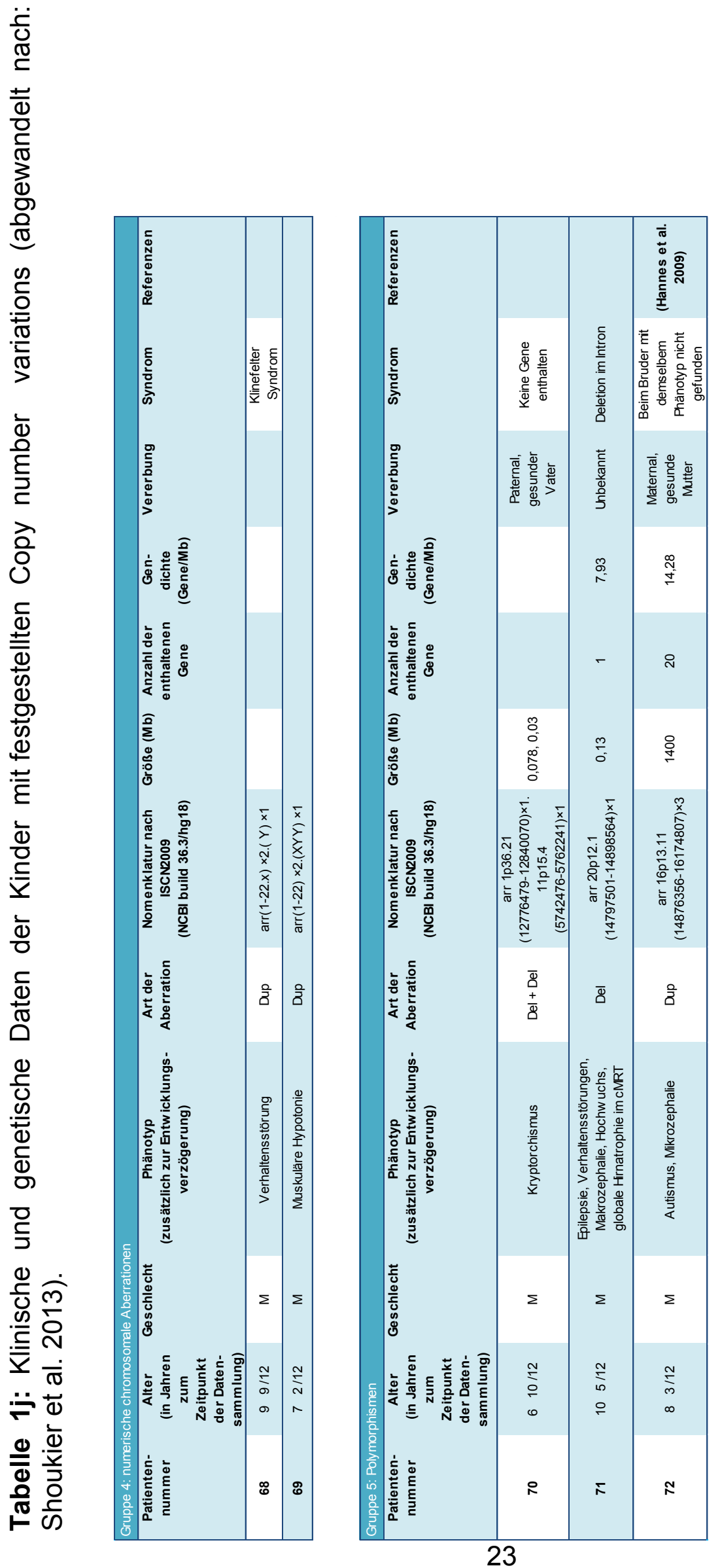

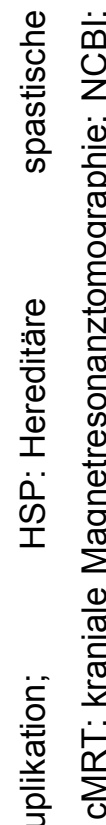

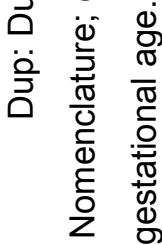

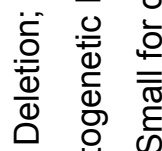

㐫

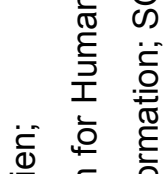

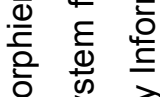

है के वे

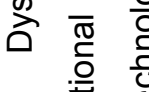

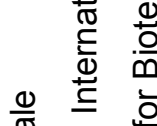

$\begin{array}{ll}\frac{0}{\pi} & \leq \\ \frac{N}{N} & \bigcup \\ \frac{\pi}{0} & \underline{0}\end{array}$

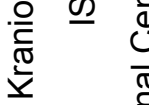

芒苍高 
Die detektierten chromosomalen Veränderungen haben Ausdehnungen zwischen 0,065 und 78,33 Mb. Die meisten CNVs haben dabei eine Größe von 1-5 Mb, was $42 \%$ entspricht. $37 \%$ aller strukturellen festgestellten Auffälligkeiten sind kleiner als $1 \mathrm{Mb}$, und $13 \%$ der Befunde befinden sich größenmäßig zwischen 5-10 Mb. Ebenfalls bei $13 \%$ waren die CNVs größer $10 \mathrm{Mb}$.

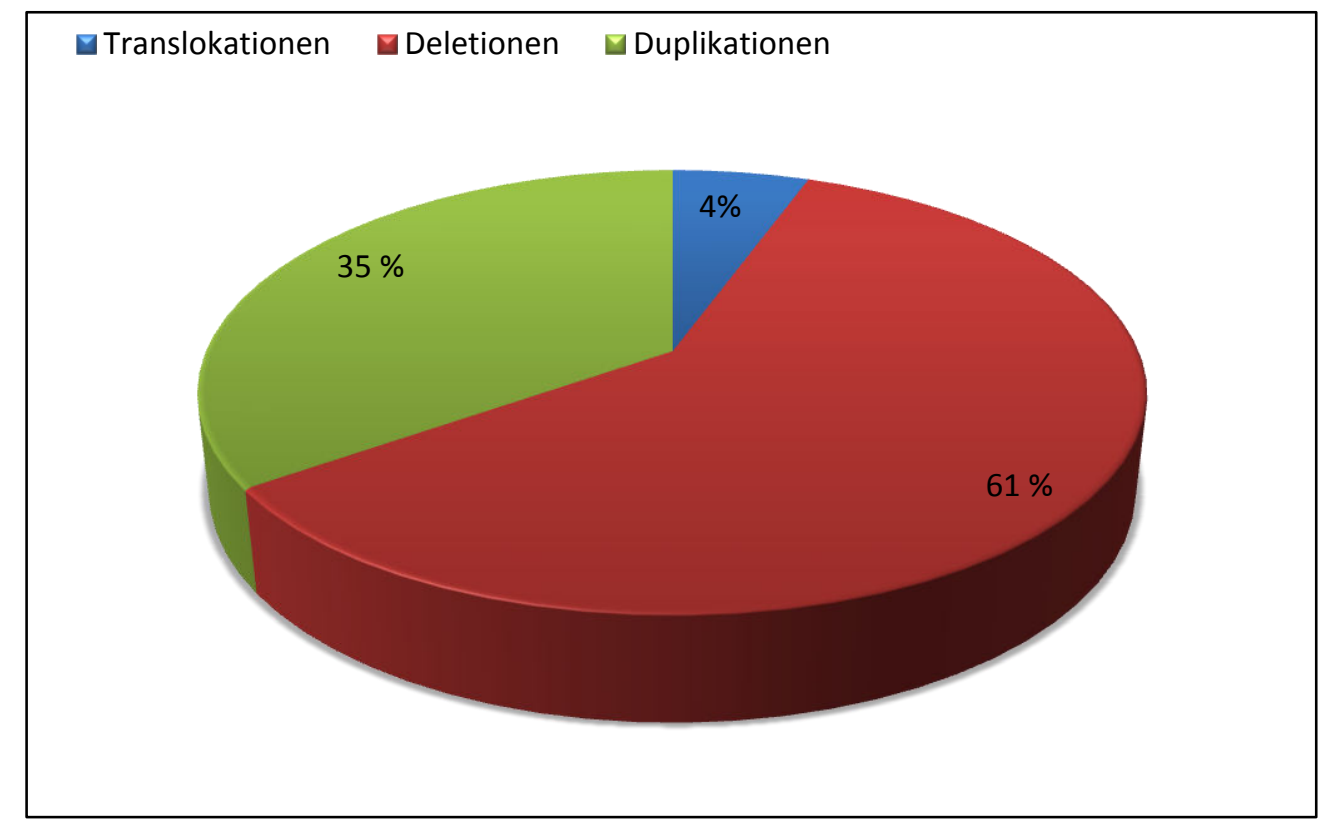

Abbildung 6: Häufigkeiten der aufgetretenen Duplikationen, Deletionen und Translokationen.

Bei den Patienten, die vor der Array-CGH bereits eine konventionelle Chromosomenanalyse erhalten haben, sind die beiden größten strukturellen Veränderungen, die trotzdem unentdeckt blieben, eine 10,8 Mb große Deletion (Patient 48) sowie eine unbalancierte Translokation ( $\mathrm{t} 5 ; 9)$, die eine Deletion von 12,2 Mb und eine Duplikation einer Größe von 10,8 $\mathrm{Mb}$ beinhaltet (Patient 45).

Die konventionelle Karyotypisierung hatte bei diesen Kindern mit einem Bandenlevel von 450-500 Mb bereits im Vorfeld stattgefunden. Bei den strukturellen Chromosomenstörungen (Gruppe 1 - 3) lassen sich in 72 \% der Fälle Aussagen über die Vererbung treffen, da bei 48 der 67 Patienten ebenfalls Untersuchungen bei Eltern und Geschwistern durchgeführt wurden. Bei 25 Kindern konnte die chromosomale Veränderung bei den Eltern nicht 
festgestellt werden und so als de-novo eingestuft werden. Bei 23 Kindern wiederum konnte dieselbe Veränderung ebenfalls bei einem Elternteil gefunden werden. Der Großteil der Eltern, bei denen ebenfalls Mutationen gefunden werden konnten, war jedoch gesund, nur bei den Patienten 15, 49 und 54 war der betroffene Elternteil ebenfalls von einer Intelligenzminderung betroffen. Maternale Vererbung trat mit 70 \% (16/23) der Fälle weitaus häufiger auf als paternale Vererbung (30\%, 7/23). Zu bemerken ist außerdem, dass alle neun Fälle von vererbten CNVs klarer klinischer Relevanz mütterlichen Ursprungs waren, wohingegen paternale Vererbung hauptsächlich bei den Fällen unklarer Genese zu finden war.

\subsection{Klinische Daten}

Bei der Auswertung der klinischen Daten wurden zwei Gruppen miteinander verglichen:

Vergleichsgruppe 1: Patienten mit chromosomalen Mikrodeletions- und duplikationssyndromen und Translokationen klarer klinischer Relevanz (Gruppe 1 und 2, n=45 Kinder)

Vergleichsgruppe 2: Kinder mit unauffälligen Array-CGH-Befund $(n=270)$.

Die Auswertung der klinischen Daten der beiden Gruppen ergab Folgendes (Abb. 7a, b): Bei $46,7 \%$ der Kinder mit phänotypisch kausalen CNVs wurden kongenitale Anomalien gefunden, in der Gruppe ohne Auffälligkeiten war dies bei 27 \% der Kinder der Fall. Es zeigt sich, dass kongenitale Anomalien bei Kindern mit klinisch relevanten CNVs signifikant häufiger auftreten ( $p$-Wert 0,003). Herzfehler (z.B. Ventrikelseptumdefekt, Atriumseptumdefekt) waren die häufigsten aufgetretenen Fehlbildungen, 22,2 \% der Kinder aus den Gruppen 1 und 2 und 8,9\% aus der Vergleichsgruppe waren davon betroffen. Somit traten auch Herzfehler signifikant häufiger bei Kindern mit klinisch relevanten CNVs auf im Vergleich zu Kindern mit normaler Array-CGH (p-Wert 0,008). Eine symptomatische Epilepsie wurde bei $28,9 \%$ der Kinder mit auffälligem Array-Befund und bei 35,9\% mit negativem Befund diagnostiziert. 


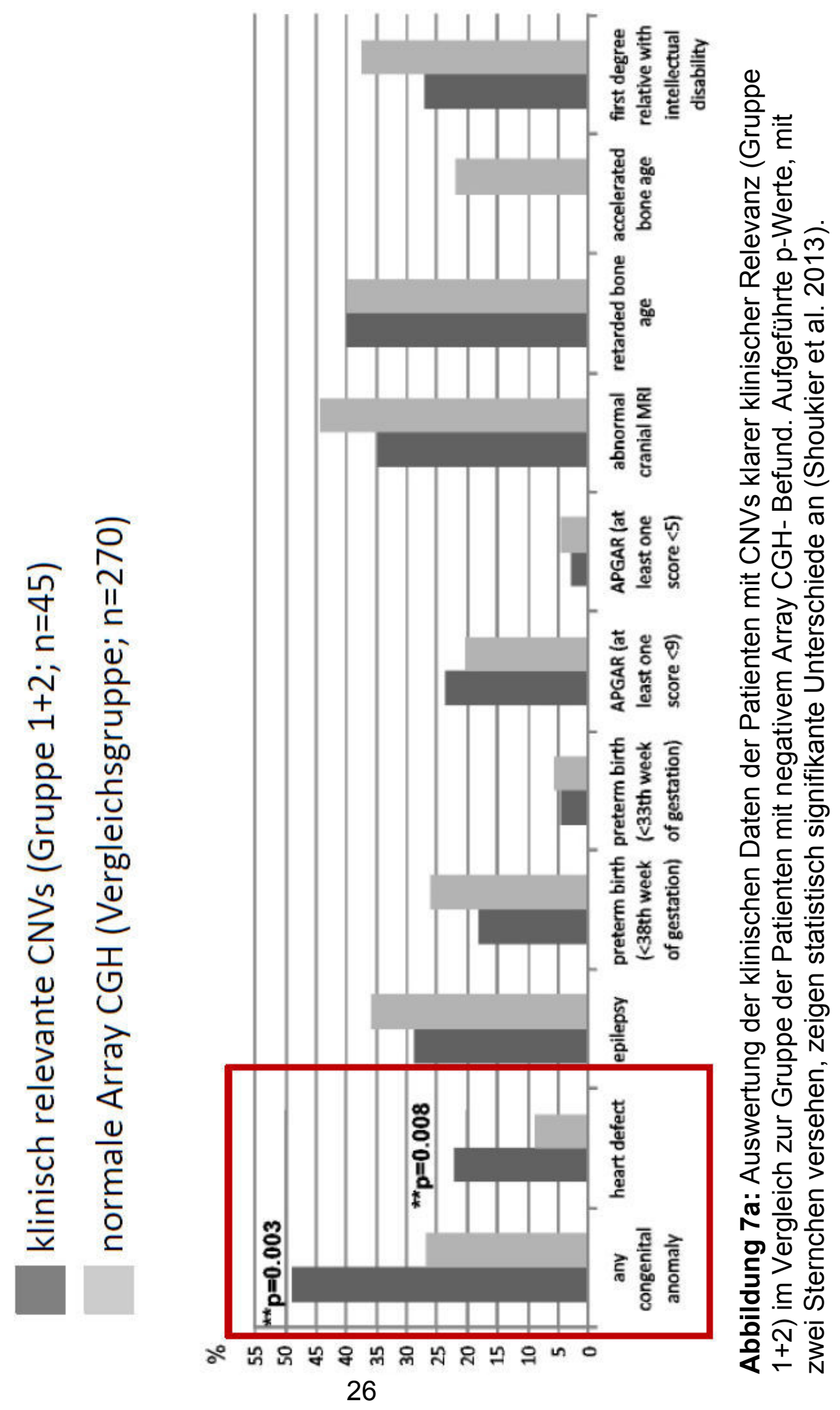




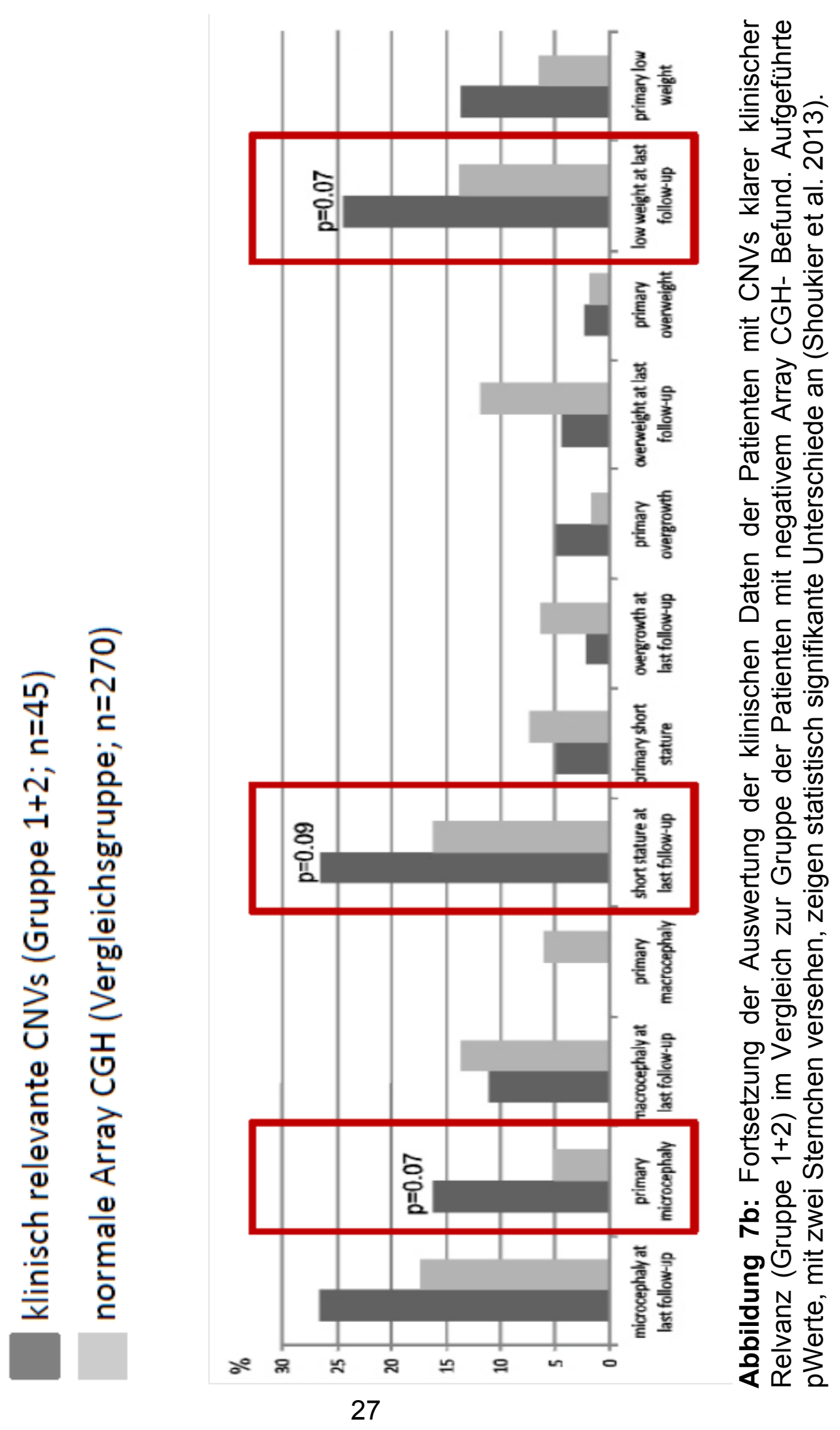


Frühgeburtlichkeit mit einem Geburtstermin vor der 38. SSW war bei $17,1 \%$ aus Vergleichsgruppe 1 und 26,1\% aus Vergleichsgruppe 2 der Fall, wohingegen eine Frühgeburt noch vor Ende der 33. SSW) in Vergleichsgruppe 1 nur bei 5,7 \% und in Vergleichsgruppe 2 bei $5,6 \%$ auftrat.

APGAR-Werte kleiner neun wurden bei $26 \%$ der Kinder mit klinisch relevanten CNVs und 20,4\% der Patienten mit negativem Array-CGH-Befund erhoben. APGAR-Werte kleiner fünf traten mit 3,7\% in Vergleichsgruppe 1 und 4,6\% in Vergleichsgruppe 2 deutlich seltener auf. Während bei 40 \% beider Vergleichsgruppen ein retardiertes Knochenalter festgestellt werden konnte, trat ein akzeleriertes Knochenalter nur bei Vergleichsgruppe 2 auf (22,5\%). Bezüglich der Körpermaße bei der Geburt sowie der aktuell vorliegenden Maße treten primäre Mikrozephalie mit 16,7\% (Vergleichsgruppe 2: 5,2 \%), Kleinwuchs zum Zeitpunkt der letzten Kontrolle mit 26,6\% (Vergleichsgruppe 2:16,2 \%) als auch Untergewicht bei der letzten Kontrolluntersuchung mit 24,4\% bei Gruppe $1+2$ (Vergleichsgruppe 2: 13,8 \%) im Vergleich zur Gruppe mit negativem Array-CGH-Befund häufiger auf. Diese Unterschiede erreichen jedoch mit einem p-Wert von 0,07 für primäre Mikrozephalie, einem p-Wert von 0,09 für Kleinwuchs zum letzten dokumentierten Zeitpunkt sowie einem p-Wert von 0,07 für Untergewicht bei der letzten Untersuchung kein statistisch signifikantes Niveau. Eine primäre Makrozephalie hingegen lag bei keinem der Kinder aus Vergleichsgruppe 1 und nur bei 6,1\% aus Vergleichsgruppe 2 vor. Primärer Kleinwuchs hatte in Gruppe $1+2$ eine Prävalenz von 6,3\% und im Vergleich dazu in der Vergleichsgruppe 2 ein Vorkommen von 7,4\%. Epilepsie, primäre Makrozephalie und Hochwuchs bei der letzten Kontrolle traten also durchaus häufiger in der Gruppe der Patienten mit negativem Array-CGH-Befund auf; diese Unterschiede sind jedoch nicht von statistischer Signifikanz.

Die fazialen Dysmorphien einiger Patienten mit durch Array-CGH festgestellten copy number variations sowohl klarer als auch unklarer Relevanz sind in Abbildung $\mathbf{8}$ dargestellt. 


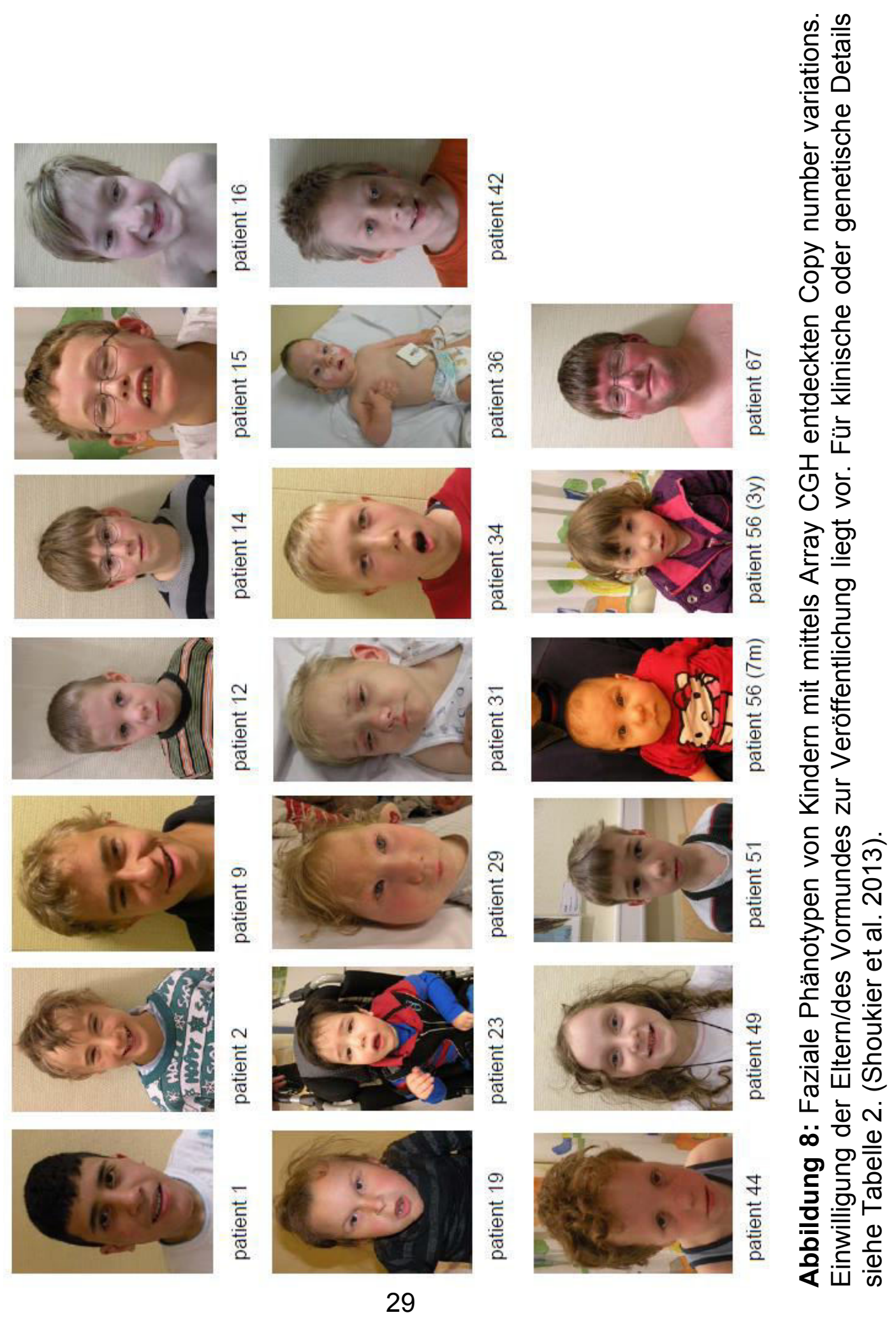




\section{Diskussion}

In unserer Studie wurden 342 Kinder mit Entwicklungsstörung oder Intelligenzminderung mittels Array-CGH untersucht. Bei 72 von 342 Kindern (21,1\%) wurden chromosomale Kopiezahlvarianten (copy number variations) gefunden. Mikrodeletionen, Mikroduplikationen sowie unbalancierte Translokationen eindeutiger klinischer Relevanz wurden in nahezu zwei Dritteln der auffälligen Befunde (45/72) festgestellt (Gruppen 1 und 2, Abbildung 5). Somit war bei 45 von 342 Patienten (13,2\%) eine Ursachenklärung der Entwicklungsverzögerung bzw. Intelligenzminderung möglich. Hingegen wurden bei $6,4 \%$ der Kinder (Gruppe 3, Abbildung 5), also 22 von insgesamt 342, CNVs bisher unklarer klinischer Relevanz ermittelt. Es handelte sich um 13 Duplikationen sowie 9 Deletionen, die entweder bisher nicht in der Literatur beschrieben sind oder von einem gesunden Elternteil vererbt wurden.

Dieser Anteil von 13,2\% klinisch relevanter CNVs ist vergleichbar mit der Array-CGHDetektionsrate zweier großer Studien, bei denen dieselbe Array-CGH-Technologie verwendet wurde. In der Studie von Cooper et al. (2011) wurden 15.767 Kinder mittels ArrayCGH untersucht und bei 14,2\% konnten pathologische copy number variations festgestellt werden.

In einer anderen Studie konnten bei $14,7 \%$ der untersuchten Kinder signifikante CNVs ermittelt werden (Kaminsky et al. 2011). Auch in weiteren Studien wurden immer wieder ähnliche Detektionsraten nachgewiesen (D`Arrigo et al. 2015, Coutton et al. 2015).

Der überwiegende Teil der in unserer Studie aufgefundenen Chromosomenaberrationen war interstitiell, nur $5 \%$ der CNVs ließen sich in telomeren Regionen finden. Ein Subtelomerscreening, das ausschließlich subtelomere Chromosomenaberrationen detektiert, wäre demnach in vielen Fällen ergebnislos geblieben. Die deutlich höhere Detektionsrate der Array-CGH hat den Einsatz des Subtelomerscreenings in der Abklärung von Entwicklungsstörungen und Intelligenzminderungen daher obsolet gemacht.

42 \% der chromosomalen Veränderungen hatten eine Größe von 1 - 5 Mb, 37 \% der Aberrationen waren sogar kleiner als $1 \mathrm{Mb}$. Solch kleine CNVs sind durch die konventionelle Chromosomenanalyse kaum zu erkennen, da sich die Auflösung einer klassischen Chromosomenanalyse zwischen 5-10 Mb bewegt (Basel-Vanagaite 2008). Zusätzlich zur Auf- 
lösung der verwendeten Array-CGH-Plattform scheint die Selektion der Patienten den größten Einfluss auf die Detektionsrate für klinisch relevante Chromosomenstörungen zu haben. Unsere Kohorte beinhaltet einen großen Teil an Kindern, die bereits zahlreiche diagnostische Verfahren zur Ursachenklärung ihrer Intelligenzminderung durchlaufen haben, so dass zuvor bereits viele andere, nicht genetische Ursachen ausgeschlossen werden

konnten. Alle Kinder hatten Routineblutuntersuchungen, metabolische Screening-Tests, ebenso wie Untersuchungen der Aminosäuren im Blutplasma und organischer Säuren im Urin. Bei $80 \%$ der Patienten wurde der Liquor cerebrospinalis untersucht und bei $70 \%$ aller Kinder wurde eine konventionelle Chromosomenanalyse, meist einschließlich Subtelomerscreening, durchgeführt, bevor die Untersuchung mittels Array-CGH durchgeführt wurde.

Die statistische Auswertung der klinischen Daten zeigt, dass kongenitale Anomalien, insbesondere Herzfehler, signifikant häufiger bei den Kindern auftraten, die klinisch relevante CNVs, also chromosomale Mikrodeletionen oder -duplikationen sowie Translokationen klarer klinischer Bedeutung hatten, im Vergleich zu Kindern mit unauffälligem Array-CGHBefund. Darüber hinaus sind klinische Kriterien wie primäre Mikrozephalie, Kleinwuchs und Untergewicht bei der letzten dokumentierten Kontrolluntersuchung Indikatoren für eine höhere Wahrscheinlichkeit einer klinisch relevanten Chromosomenaberration, obwohl keine statistische Signifikanz erreicht werden konnte. Bei Kindern mit diesen phänotypischen Auffälligkeiten ist demnach die Durchführung einer Array-CGH besonders sinnvoll und hat eine gute Erfolgsrate.

Das gehäufte Vorkommen von kongenitalen Anomalien bei Patienten mit klinisch signifikanten Mutationen konnte bereits in verschiedenen Studien gezeigt werden (Baris et al. 2007, Cooper et al. 2011, Lu et al. 2008). Dennoch ist das Spektrum an verschiedenen Phänotypen sehr vielfältig und auch bei Kindern mit nicht syndromaler, isolierter Intelligenzminderung oder Entwicklungsverzögerung konnten in der Array-CGH-Untersuchung ursächliche chromosomale Aberrationen gefunden werden. Eine Array-CGH-Untersuchung sollte daher nicht nur Kindern mit kongenitalen Anomalien oder phänotypischen Auffälligkeiten vorbehalten sein, sondern wird mittlerweile bei allen Kindern mit unklarer Entwicklungsstörung oder Intelligenzminderung empfohlen (Miller et al. 2010). 
Die klinische Relevanz von $31 \%$ der festgestellten CNVs (Gruppe 3) ist jedoch leider auch beim derzeitigen Kenntnisstand unklar.

Hierfür gibt es folgende Gründe:

1. Die chromosomale Aberration ist bisher unbekannt, und es wurde noch nichts darüber veröffentlicht.

2. Die Mutation wurde von einem gesunden Elternteil vererbt, was eine Zuordnung zu pathologischen CNVs auf Grund unvollständiger Penetranz oder das eventuelle Vorliegen eines familiären Polymorphismus ausschließt.

Vermutlich wird sich die Relevanz solcher bislang unklarer CNVs in wenigen Jahren eindeutig zuordnen lassen. Voraussetzung hierfür sind die Sammlung weiterer phänotypischer und genetischer Daten der Array-CGH in Datenbanken sowie die Publikation dieser Daten. Mit der vorliegenden Arbeit soll hierzu beigetragen werden. 


\section{Ausblick}

Auch nach Abschluss unserer Studie bleibt die Array-CGH von großer Relevanz und gilt nach wie vor als Basisdiagnostik in der genetischen Abklärung von Kindern mit Dysmorphie- und Retardierungs-Syndromen unklarer Genese (O‘Byrne et al. 2016, Dan et al. 2014).

Mittlerweile gibt es aber auch eine Vielzahl neuer Verfahren zur DNA-Sequenzierung, die unter dem Begriff next generation sequencing (NGS) zusammengefasst werden. Diese Untersuchungsmethoden haben alle die Parallelisierung des Sequenzierungsprozesses in sehr kleinen Volumina mit daraus resultierendem hohen Durchsatz gemeinsam (Bettecken et al. 2014). Es können sowohl das gesamte Genom (whole genome sequencing) als auch nur die gencodierende Bereiche sequenziert werden (whole exome sequencing) (Hempel et al. 2011). Auch die Untersuchung der DNA ausgewählter Gene, die spezifisch für einen bestimmten Phänotyp sind, ist möglich. Dafür werden sogenannte Gen-Panels zusammengestellt, die etwa 20-200 krankheitsspezifische Gene enthalten, die parallel sequenziert werden können (Zirn 2012, Hempel et al. 2011, Bettecken et al. 2014). Auch CNVs werden bei diesen Untersuchungsmethoden miterfasst. Mittels next generation sequencing lässt sich eine Auflösungen von nur einem Basenpaar erzielen und somit Punktmutationen erkennen.

Derzeit können durch die neuen Hochdurchsatz-Sequenzierungs-Verfahren bei vielen Kindern mit bislang unklaren Dysmorphie- und Retardierungssyndromen Diagnosen gestellt werden. Allerdings ist auch die Rate an unklaren Befunden erhöht. Es wird aktuell an internationalen Datenbanken zur Interpretation bislang unklarer Varianten gearbeitet (Zirn 2012, Bettecken et al. 2014). Außerdem entstehen durch die große Menge anfallender Daten dieser Verfahren auch neue ethische und rechtliche Konflikte. Es muss insbesondere festgelegt werden, wie die erhobenen Daten sicher gespeichert und in welchem Maße weitergegeben werden können. Außerdem stellt sich die Frage, wie mit Zufallsinformationen und Nebenbefunden umgegangen werden soll und wie und in welchem Maße die Patienten künftig aufgeklärt werden müssen (Deutscher Ethikrat 2013). 


\section{Zusammenfassung}

In den letzten Jahren hat sich die Array-CGH als primäres genetisches ScreeningVerfahren in der Abklärung von Entwicklungsstörungen und geistigen Behinderungen durchgesetzt. In der vorliegenden Promotionsarbeit wurden zwei Ziele verfolgt: Zum einen sollte das phänotypische Spektrum klinisch relevanter chromosomaler Kopiezahländerungen erweitert werden, zum anderen sollten klinische Kriterien erarbeitet werden, die mit einer erhöhten Wahrscheinlichkeit für das Auffinden eines klinisch relevanten CNVs einhergehen.

Es wurde eine retrospektive Analyse der klinischen Daten und der Array-CGH-Befunde von 342 Kindern mit Entwicklungsstörungen oder Intelligenzminderung durchgeführt. Die klinischen Parameter der Kinder mit in der Array-CGH detektierten und klinisch relevanten CNVs wurden dabei mit den Daten der Kinder mit unauffälligem Array-CGH-Befund verglichen.

Bei 45 von 342 (13,2\%) Kindern wurden klinisch relevante Kopiezahländerungen (chromosomale Mikrodeletionen und Mikroduplikationen sowie unbalancierte Translokationen) aufgefunden und eine Genotyp-Phänotyp-Korrelation durchgeführt. Bei der Auswertung der klinischen Daten zeigten sich bei Kindern mit klinisch relevanten CNVs signifikant häufiger angeborene Fehlbildungen, insbesondere Herzfehler. Deutlich häufiger wiesen diese Kinder zudem eine primäre Mikrozephalie, einen sekundären Kleinwuchs und/oder eine Dystrophie auf. Bei Kindern mit bislang unklarer Entwicklungsstörung oder geistiger Behinderung, die diese klinischen Merkmale aufweisen, besteht demnach eine höhere Wahrscheinlichkeit für die Detektion einer klinisch relevanten Chromosomenstörung in der Array-CGH. 


\section{Literaturverzeichnis}

American Psychiatric Association (2000):

Diagnostic and Statistical Manual of Mental disorders. DSM-IV-TR. 4. Auflage, Text Revision

Baris HN, Tan WH, Kimonis VE, Irons MB (2007):

Diagnostic utility of array-based comperative genomic hybridization in a clinical setting. Am J Med Genet A 143A, 2523-2533

Basel-Vanagaite L (2008):

Clinical Approaches to Genetic Mental Retardation. Isr Med Assoc J 10, 821-826

Bedeschi MF, Novelli A, Bernardini L, Parazzini C, Bianchi V, Torres B, Natacci F, Giuffrida MG, Ficarazzi P, Dallapiccola B (2008):

Association of syndromic mental retardation with an Xq12q13.1 duplication encompassing the oligophrenin 1 gene. Am J Med Genet A 146A, 1718-1724

Berg JS, Brunetti-Pierri N, Peters SU, Kang SH, Fong CT, Salamone J, Freedenberg D, Hannig VL, Prock LA, Miller DT (2007):

Speech delay and autism spectrum behaviours are frequently associated with duplication of the 7q11.23 Williams-Beuren syndrome region. Genet Med 9, 427-441

Bettecken T, Pfeufer A, Sudbrak R, Siddiqui R, Franke A, Wienker TF, Krawczak M (2014): Next Generation Sequencing in der diagnostischen Praxis. Med Gen 26, 21-27

Caliebe A, Kroes HY, van der Smagt JJ, Martin-Subero Jl, Tönnies H, van 't Slot R, Nievelstein RA, Muhle H, Stephani U, Alfke K (2010):

Four patients with speech delay, seizures and variable corpus callosum thickness sharing a $0.440 \mathrm{Mb}$ deletion in region 1944 containing the HNRPU gene. Eur J Med Genet 53, 179185

Chabrol B, Martens K, Meulemans S, Cano A, Jaeken J, Matthijs G, Creemers JW (2008): Deletion of C20rf34, PREPL and SLC3A1 causes atypical hypotonia-cystinuria syndrome. J Med Genet 45, 314-318 
Chelly J, Khelfaoui M, Francis F, Cherif B, Bienvenu T (2006):

Genetics and pathophysiology of mental retardation. Eur J Hum Genet 14, 701-713

Ching MS, Shen Y, Tan WH, Jeste SS, Morrow EM, Chen X, Mukaddes NM, Yoo SY, Hanson

E, Hundley R. (2010):

Deletions of NRXN1 (neurexin-1) predispose to a wide spectrum of developmental disorders. Am J Med Genet B Neuropsychiatr Genet 153B, 938-947

Collins VR, Muggli EE, Riley M, Palma S, Halliday JL (2008):

Is Down syndrome a disappearing birth defect? J Pediatr 152, 20-24

Cooper GM, Coe BP, Girirajan S, Rosenfeld JA, Vu TH, Baker C, Williams C, Stalker H, Hamid R, Hannig V et al. (2011):

A copy number variation morbidity map of developmental delay. Nat Genet $43,838-846$

Costeff H, Cohen BE, Weller L (1972):

Parental consanguinity among Israeli mental retardates. Acta Paediatr Scand 61, 452-458

Coutton C, Dieterich K, Satre V, Vieville G, Amblard F, David M, Cans C, Jouk P-S, Devillard $F(2015)$ :

Array- CGH in children with mild intellectual disability: a population- based study. Eur J

Pediatr $174,75-83$

Croen LA, Grether JK, Selvin S (2001):

The epidemiology of mental retardation of unknown cause. Pediatrics 107, E86

Curry CJ, Stevenson RE, Aughton D, Byrne J, Carey JC, Cassidy S, Cunniff C, Graham JM Jr, Jones MC, Kaback MM (1997):

Evaluation of mental reatardation: recommendations of a consensus conference: American College of Medical Genetics. Am J Med Genet 72, 468-477

D’Arigo S, Gavazzi F, Alfei E, Zuffardi O, Montomoli C, Corso B, Buzzi E, Sciacca FL, Bulgheroni S, Riva D (2015):

The diagnostic yield of array comperative genomic hybridization is high regardless of severity of intellectual disability/developmental delay in children. J Child Neurol. 2015 Oct 28. pii: 0883073815613562 . [Epub ahead of print] 
Dan B, Baxter P (2014):

Paediatric neurology: a year of DNA technology. Lancet Neurol. 2014 Jan;13(1):16-8. doi: $10.1016 / S 1474-4422(13) 70283-7$

De Ravel T, Devriendt K, Fryns JP, Vermeesch JR (2007):

What's new in karyotyping? The move towards array comparative genomic hybridization (CGH). Eur J Pediatr 166, 637-643

Deutsches Institut für Medizinische Dokumentation und Information (2012) [Hg.]: ICD-10-GM. Systematisches Verzeichnis. Internationale statistische Klassifikation der Krankheiten und verwandter Gesundheitsprobleme. 10. Revision: Online verfügbar unter: http://www.icd-code.de/ [Stand: 27.03.2012]

Deutscher Ethikrat (2013) [Hg.]:

Die Zukunft der genetischen Diagnostik- von der Forschung in die klinische Anwendung. Online verfügbar unter: http://www.ethikrat.org/dateien/pdf/stellungnahme-zukunft-dergenetischen-diagnostik.pdf [Stand: 30.03 .2016$]$.

Deutsche Gesellschaft für Kinder- und Jugendpsychiatrie, Psychosomatik und Psychotherapie (2014):

S2k Praxisleitlinie Intelligenzminderung. Online verfügbar unter:

http://www.awmf.org/uploads/tx_szleitlinien/028042I_S2k_Intelligenzminderung_201412.pdf [Stand: 31.03.2016]

Dolan M, Mendelsohn NJ, Pierpont ME, Schimmenti LA, Berry SA, Hirsch B (2010): A novel microdeletion/microdupliation syndrome of 19p13.13. Genet Med 12, 503-511

Durkin MS (2002):

The epidemiology of developmental disabilities in low-income countries. Ment Retard Dev Disabil Res Rev $\underline{8}$, 206-211

Durkin MS, Hasan ZM, Hasan KZ (1998):

Prevalence and correlates of mental retardation among children in Karachi, Pakistan. Am J Epidemiol 147, 281-288 
Durkin MS, Khan NZ, Davidson LL, Hug S, Munir S, Rasul E, Zaman SS (2000):

Prenatal and postnatal risk factors for mental retardation among children in Bangladesh. Am J Epidemiol 152, 1024-1033

Feuk L, Carson AR, Scherer SW (2006):

Structural variation in the human genome. Nat Rev Genet 7, 85-97.

Frints SG, Schrander-Stumpel CT, Schoenmakers EF, Engelen JJ, Reekers AB, Van den Neucker AM, Smeets E, Devlieger H, Fryns JP (1998):

Strong variable clinical presentation in 3 patients with $7 q$ terminal deletion. Genet Couns 9, 5-14

Gillessen-Kaesbach G, Sieber R (2012):

Stellenwert der Array-basierten molekularen Karyotypisierung in der Humangenetik. Med Gen 24, 84-85

Giorda R, Bonaglia MC, Beri S, Fichera M, Novara F, Magini P, Urquhart J, Sharkey FH, Zucca C, Grasso R (2009):

Complex segmental duplications mediate a recurrent $\operatorname{dup}(\mathrm{X})(\mathrm{p} 11.22-\mathrm{p} 11.23)$ associated with mental retardation, speech delay, and EEG anomalies in males and females. Am J Hum Genet $85,394-400$

Hannes FD, Sharp AJ, Mefford HC, de Ravel T, Ruivenkamp CA, Breuning MH, Fryns JP, Devriendt K, Van Buggenhout G, Vogels A (2009):

Recurrent reciprocal deletions and duplications of 16p13.11: the deletion is a risk factor for MR/MCA while the duplication may be a rare benign variant. J Med Genet 46, 223-232

Heinzen EL, Radtke RA, Urban TJ, Cavalleri GL, Depondt C, Need AC, Walley NM, Nicoletti P, Ge D, Catarino CB (2010):

Rare deletions at 16p13.11 predispose to a diverse spectrum of sporadic epilepsy syndromes. Am J Hum Genet 86 , 707-718

Hempel M, Haack TB, Eck S, Prokisch H (2011):

“Next Generation Sequencing” Neuer Zugang zur molekularen Aufklärung und Diagnostik von Stoffwechseldefekten. Monatsschr Kinderheilkd 159, 827-833 
Hilhorst-Hofstee Y, Tumer Z, Born P, Knijnenburg J, Hansson K, Yatawara V, Steensberg J, Ullmann R, Arkesteijn G, Tommerup N (2009):

Molecular characterization of two patients with de novo interstitial deletions in 4q22-q24. Am J Med Genet A 149A, 1830-1833

lafrate AJ, Feuk L, Rivera MN, Listewnik ML, Donahoe PK, Qi Y, Scherer SW, Lee C (2004): Detection of large-scale variation in the human genome. Nat Genet 36, 941-951

Itsara A, Cooper GM, Baker C, Girirajan S, Li J, Absher D, Krauss RM, Myers RM, Ridker PM, Chasman DI (2009):

Population analysis of large copy number variants and hotspots of human genetic disease. Am J Hum Genet 84, 148-161

Kaminsky EB, Kaul V, Paschall J, Church DM, Bunke B, Kunig D, Moreno-De-Luca D, Moreno-De-Luca A, Mulle JG, Warren ST (2011):

An evidence-based approach to establish the functional and clinical significance of copy number variants in intellectual and development disabilities. Genet Med 13, 777-784

Klein HG, Rost I (2012):

Zentrum für Humangenetik und Laboratoriumsmedizin. Laborinformation 10. Online verfügbar unter: http://www.medizinische-

genetik.de/fileadmin/Image_Archive/Laborinfos/laborinfo10.pdf (Stand: 27.03.2012)

Leonard H, Wen X (2002):

The epidemiology of mental retardation: challenges and opportunities in the new millennium. Ment Retard Dev Disabil Res Rev $\underline{8}, 117-34$

Lespinasse J, Gimelli S, Bena F, Antonarakis SE, Ansermet F, Paoloni-Giacobino A (2009): Characterization of an interstitial deletion 6q13-q14.1 in a female with mild mental retardation, language delay and minor dysmorphisms. Eur J Med Genet 52, 49-52

Locke DP, Sharp AJ, McCarroll SA, McGrath SD, Newman TL, Cheng Z, Schwartz S, Albertson DG, Pinkel D, Altshuler DM (2006):

Linkage disequilibrium and heritability of copy-number polymorphisms within duplicated regions of the human genome. Am J Hum Genet 79, 275-290 
Lorentz CP, Jalal SM, Thompson DM, Babovic-Vusanovic D (2002):

Mosaic $r(13)$ resulting in large deletion of chromosome $13 q$ in a newborn female with multiple congenital anomalies. Am J Med Genet 111, 61-67

Lu XY, Phung MT, Shaw CA, Pham K, Neil SE, Patel A, Sahoo T, Bacino CA, Stankiewicz P, Kang SH (2008):

Genomic imbalances in neonates with birth defects: high detection rates by using chromosomal microarray analysis. Pediatrics 122, 1310-1318

Magariello A, Muglia M, Patitucci A, Ungaro C, Mazzei R, Gabriele AL, Sprovieri T, Citrigno L, Conforti FL, Liguori M (2010):

Mutation analysis of the SPG4 gene in Italian patients with pure and complicated forms of spastic paraplegia. J Neurol Sci $288,96-100$

Manning M, Hudgins L (2010):

Array-based technology and recommendations for utilization in medical genetics practice for detectionof chromosomal abnormalities. Genet Med 12, 742-745

Mefford HC, Sharp AJ, Baker C, Itsara A, Jiang Z, Buysse K, Huang S, Maloney VK, Crolla JA, Baralle D et al. (2008):

Recurrent rearrangements of chromosome 1q21.1 and variable pediatric phenotypes. $\mathrm{N}$ Engl J Med 359, 1685-1699

Mefford HC, Batshaw ML, Hoffman EP (2012):

Genomics, Intellectual Disability, and Autism. N Engl J Med 366, 733-743

Miclea D, Peca L, Cuzmici Z, Pop IV (2015):

Genetic testing in patients with global developmental delay/ intellectual disabilities. A review. Clujul Med 88, 288-292

Miller DT, Adam MP, Aradhya S, Biesecker LG, Brothman AR, Carter NP, Church DM, Crolla JA, Eichler EE, Epstein CJ et al. (2010):

Consensus statement: chromosomal microarray is a first-tier clinical diagnostic test for individuals with development disabilities or congenital anomalies. Am J Hum Genet 86, $749-764$ 
Moog U, Kutsche K, Kortum F, Chilian B, Bierhals T, Apeshiotis N, Balg S, Chassaing N, Coubes C, Das S et al. (2011):

Phenotypic spectrum associated with CASK loss-of-function mutations. J Med Genet 48, 741-751

Nagamani SC, Erez A, Shen J, Li C, Roeder E, Cox S, Karaviti L, Pearson M, Kang SH, Sahoo T et al. (2010):

Clinical spectrum associated with recurrent genomic rearrangements in chromosome 17q12. Eur J Hum Genet 18, 278-284

Nazer HJAR, Cifuentes OL (2006):

Increasing rates of Down Syndrome among newborns in Chile from 1972 to 2005. Rev Med Chil 134, 1549-1557

Neveling K, Hoischen A (2012):

Exom Sequenzierung zur Identifizierung von Krankheitsgenen. Med Gen 24, 4-11

O’Byrne JJ, Lynch SA, Treacy EP, King MD, Betts DR, Mayne PD, Sharif F (2016):

Unexplained developmental delay/learning disability: guidelines for best practice protocol for first line assessment ans genetic/ metabolic/radiological investigations. Ir J Med Sci $185,241-248$

Oneda B, Rauch A (2012):

Molekulare in der Diagnostik neurokognitiver Entwicklungsstörungen. Med Gen 24, 94-98

Rauch A (2010):

Genetik der mentalen Retardierung und Intelligenzminderung. Pädiatrie 610, 10-14

Rauch A, Dorr HG (2007):

Chromosome 5q subtelomeric deletion syndrome. Am J Med Genet C Semin Med Genet 145 C, $372-376$

Rauch A, Hoyer J, Guth S, Zweier C, Kraus C, Becker C, Zenker M, Huffmeier U, Thiel C, Ruschendorf F et al (2006):

Diagnostic yield of various genetic approaches in patients with unexplained development delay or mental retardation. Am J Med Genet A 140, 2063-2074 
Redon R, Ishikawa S, Fitch KR, Feuk L, Perry GH, Andrews TD, Fiegler H, Shapero MH, Carson AR, Chen W et al. (2006):

Global variation in copy number in the human genome. Nature $444,444-454$

Robertson SP, O’Day K, Bankier A (1998):

The 4q-syndrome: delineation of the minimal critical region within band 4q31. Clin Genet $53,70-73$

Roeleveld N, Zielhuis GA, Gabreëls F (1997):

The prevalence of mental retardation: A critical review of recent literature. Dev Med Child Neurol 39, 125-132

Ropers HH (2008):

Genetics of intellectual disability. Curr Opin Genet \& Dev 18, 241-250

Ropers HH, Hamel BC (2005):

X-linked mental retardation. Nat Rev Genet $\underline{6}$, 46-57

Salvador-Carulla L, Bertelli M (2008):

„Mental Retardation“ or „Intellectual disability“: time for a conceptual change. Psychopathology 41, 10-16

Sebat J, Lakshmi B, Troge J, Alexander J, Young J, Lundin P, Månér S, Massa H, Walker M, Chi $M$ et al. (2004):

Large-scale copy number polymorphism in the human genome. Science $305,525-528$

Shoukier M, Wickert J, Schroder J, Bartels I, Auber B, Zoll B, Salinas-Riester G, Weise D, Brockmann K, Zirn B et al. (2011):

A $16 q 12$ microdeletion in a boy with severe psychomotor delay, craniofacial dysmorphism, brain and limb malformations, and a heart defect. Am J Med Genet A. 2011 Dec 2. doi: 10.1002/ajmg.a.34387 [Epub ahead of print] 
Shoukier M, Schroder J, Zoll B, Burfeind P, Freiberg C, Klinge L, Kriebel T, Lingen M, Mohr A, Brockmann K (2012):

A de novo interstitial deletion of 2p23.3-24.3 in a boy presenting with intellectual disability, overgrowth, dysmorphic features, skeletal myopathy, dilated cardiomyopathy. Am J Med Genet A 158, 429-433

Shoukier M, Klein N, Auber B, Wickert J, Schröder J, Zoll B, Burfeind P, Bartels I, Alsat E, Lingen $M$ et al. (2013):

Array CGH in patients with developmental delay or intellectual disability: are there phenotypic clues to pathogenic copy number variants? Clin Genet.83, 53-65

Spengler S, Begemann M, Eggermann K, Zerres K, Ortiz Brüchle N, Seidel H, RudnikSchöneborn S, Eggermann T (2013):

Einsatz der molekularen Karyotypisierung in der Pädiatrie. Monatsschr Kinderheilkd 161, $633-643$

Stettner GM, Shoukier M, Hoger C, Brockmann K, Auber B (2011):

Familial intellectual disability and autistic behavior caused by a small FMR2 gene deletion. Am J Med Genet A 155, 2003-2007

Striano P, Malacarne M, Cavani S, Pierluigi M, Rinaldi R, Cavaliere ML, Rinaldi MM, De Bernardo C, Coppola A, Pintaudi M et al. (2006):

Clinical phenotype and molecular characterization of $6 \mathrm{q}$ terminal deletion syndrome: five new cases. Am J Med Genet A 140, 1944-1949

Van Bokhoven H (2011):

Genetic and epigenetic networks in intellectual disabilities. Annu Rev Genet 45, 81-104

Van Karnebeek CD, Jansweijer MC, Leenders AG, Offringa M, Hennekam RC (2005): Diagnostic investigations in individuals with mental retardation: a systematic literature review of their usefullness. Eur J Hum Genet 13, 6-25

Vissers LE, De Ligt J, Gilissen C, Janssen I, Steehouwer M, de Vries P, van Lier B, Arts P, Wieskamp N, del Rosario M et al. (2010):

A de novo paradigm for mental retardation. Nat Genet 42, 1109-1112 
Willemsen MH, Kleefstra T (2014):

Making headway with genetic diagnostics of intellectual disabilities. Clin Genet $\underline{85}, 101-110$ Wischmeijer A, Magini P, Giorda R, Gnoli M, Ciccone R, Cecconi L, Franzoni E, Mazzanti L, Romeo G, Zuffardi O et al. (2011):

Olfactory receptor-related duplicons mediate a microdeletion at 11q13.2q13.4 associated with a syndromic phenotype. Mol Syndromol 1, 176-184

Zirn, B (2012):

Diagnostik bei Entwicklungsstörungen und seltenen syndromalen Erkrankungen. Kinderärztl Prax 83, 272-278 
Die Daten der vorliegenden Promotionsarbeit wurden bereits publiziert (vgl. Anhang):

Shoukier M, Klein N, Auber B, Wickert J, Schröder J, Zoll B, Burfeind P, Bartels I, Alsat EA, Lingen M, Grzmil P, Schulze S, Keyser J, Weise D, Borchers M, Hobbiebrunken E, Röbl M, Gärtner J, Brockmann K, Zirn B (2013):

Array $\mathrm{CGH}$ in patients with developmental delay or intellectual disability: are there phenotypic clues to pathogenic copy number variants? Clin Genet $83,53-65$

Ausgehend von diesen Daten ist ein Folgeprojekt im Rahmen einer weiteren Promotion entstanden. Die entsprechende Promotion wurde ebenfalls bereits eingereicht. 


\section{Danksagung}

Ich möchte mich in erster Linie bei Frau apl. Prof. Dr. med. Dr. rer. nat. Birgit Zirn für die Möglichkeit bedanken, meine Doktorarbeit über ein so interessantes Thema mit ihrer Hilfe zu verfassen. Besonders dankbar bin ich für die, nicht nur fachlich, hervorragende Betreuung. Es wurde keine Zeit und keine Mühe gescheut, mir mit gutem Rat zur Seite zu stehen und mich stets zu motivieren. Ich hätte mir keine bessere Betreuung vorstellen können, da ich zu jeder Zeit einen vertrauensvollen Ansprechpartner hatte.

Ebenfalls möchte ich mich bei Herrn Dr. med. Moneef Shoukier bedanken, der mich ebenfalls sehr gut betreut hat, stets offen für all meine Fragen und Anliegen war und von dem ich sehr viel über Humangenetik gelernt habe.

Mein Dank gilt außerdem den Leitern der beteiligten Abteilungen: Frau Prof. Dr. med. Jutta Gärtner, Direktorin der Klinik für Kinder- und Jugendmedizin der Universitätsmedizin in Göttingen, Herrn Prof. Dr. med. Dr. h.c. Wolfgang Engel, Direktor des Instituts der Humangenetik in Göttingen sowie Herrn Prof. Dr. med. Knut Brockmann, Leiter des Sozialpädiatrischen Zentrums der Klinik für Kinder- und Jugendmedizin der Universitätsmedizin Göttingen. Für die statistischen Auswertungen möchte ich mich herzlich bei Dr. Pavel Grzmil aus dem Institut für Humangenetik Göttingen bedanken.

Zu guter Letzt möchte ich den hilfsbereiten Damen von der Leitstelle und dem Sekretariat des SPZ, Frau Vogel, Frau Görlich und Frau Taugs für die Hilfe bei der Aktenrecherche danken.

Ohne diese Hilfe und Unterstützung wäre diese Arbeit niemals zustande gekommen, dafür bin ich sehr dankbar. 


\section{Lebenslauf}

Mein Name ist Nina Klein; ich wurde am 8.4.1988 in Idar-Oberstein geboren. Meine Schulausbildung begann ich 1994 auf der Grundschule Idarbachtal in Idar-Oberstein, wechselte 1998 auf das Gymnasium an der Heinzenwies und erlangte 2007 das Abitur. Von August 2007 bis August 2008 besuchte ich das Bildungsinstitut des Deutschen Roten Kreuzes in Konz, machte eine Ausbildung zur Rettungsassistentin und erwarb den Ausbildungsgrad einer Rettungssanitäterin. Vom Sommersemester 2008 bis zum Sommersemester 2010 war ich an der Georg- August- Universität in Göttingen im Studiengang Humanmedizin als Teilstudienplatzinhaberin eingeschrieben und legte dort im August 2010 das Physikum mit der Note „gut“ ab. Im Wintersemester 2010/2011 erhielt ich keinen Studienplatz für den klinischen Studienabschnitt und begann daraufhin im Dezember 2010 mit meiner Doktorarbeit an der Georg-August-Universität. Gleichzeitig arbeitete ich auf der Rettungswache des Deutschen Roten Kreuzes in Hermeskeil. Zum Sommersemester 2011 erhielt ich einen Studienplatz an der Universität des Saarlandes und nahm mein Studium im Wintersemester 2011/2012 im 1. Klinischen Semester wieder auf. Im April 2014 legte ich den schriftlichen Teil des Examens (M2) ab. Ich wechselte anschließend an die Johannes GutenbergUniversität in Mainz und absolvierte mein Praktisches Jahr von Mai 2014 bis Mai 2015 im Mutterhaus der Borromäerinnen in Trier. Dort legte ich im Mai 2015 den mündlichen Teil des Examens ab (M3) und konnte das Medizinstudium mit der Note „gut“ abschließen. Seit dem 15.06.2015 bin ich nun als Assistenzärztin der Chirurgie im Klinikum Mutterhaus der Borromäerinnen in Trier tätig. 


\section{Short Report}

\section{Array CGH in patients with developmental delay or intellectual disability: are there phenotypic clues to pathogenic copy number variants?}

Shoukier M, Klein N, Auber B, Wickert J, Schröder J, Zoll B, Burfeind P, Bartels I, Alsat EA, Lingen M, Grzmil P, Schulze S, Keyser J, Weise D, Borchers M, Hobbiebrunken E, Röbl M, Gärtner J, Brockmann K, Zirn B. Array CGH in patients with developmental delay or intellectual disability: are there phenotypic clues to pathogenic copy number variants?

Clin Genet 2012. (C) John Wiley \& Sons A/S, 2012

Array comparative genomic hybridization (array CGH) is now widely adopted as a first-tier clinical diagnostic test in individuals with unexplained developmental delay/intellectual disability (DD/ID) and congenital anomalies. Our study aimed at enlarging the phenotypic spectrum associated with clinically relevant copy number variants (CNVs) as well as delineating clinical criteria, which may help separating patients with pathogenic CNVs from those without pathogenic CNVs. We performed a retrospective review of clinical and array CGH data of 342 children with unexplained DD/ID. The phenotypic features of patients with clinically significant $\mathrm{CNV}$ were compared with those without pathogenic CNVs. Array CGH detected pathogenic CNVs in $13.2 \%$ of the patients. Congenital anomalies, especially heart defects, as well as primary microcephaly, short stature and failure to thrive were clearly more frequent in children with pathogenic CNVs compared with children with normal array CGH results. Thus, we assume that in patients with unexplained DD/ID, array CGH will more probably detect a significant CNV if any of these features is part of the patient's phenotype.

\section{Conflict of interest}

We declare that there are no conflicts of interest.

\author{
M Shoukier ${ }^{\mathrm{a}}$, N Klein ${ }^{\mathrm{a}, \mathrm{b}}$, \\ B Auber, ${ }^{a, c}$, J Wickert ${ }^{a}$, \\ J Schröder ${ }^{\mathrm{a}}$, B Zoll ${ }^{\mathrm{a}}$, \\ P Burfeind ${ }^{a}$, I Bartels ${ }^{a}$, \\ EA Alsat ${ }^{a}$, M Lingen ${ }^{b}$, \\ P Grzmil ${ }^{a, d}$, S Schulze ${ }^{b}$, \\ J Keyserb, D Weise ${ }^{\text {b }}$, \\ M Borchers ${ }^{\mathrm{b}}$, E \\ Hobbiebrunken ${ }^{b}, M_{\text {Röbl }}$, \\ J Gärtner ${ }^{b}, K$ Brockmann ${ }^{b}$ and \\ B Zirn ${ }^{b}$
}

anstitute of Human Genetics, and bepartment of Paediatrics and Paediatric Neurology, University of Göttingen, Göttingen, Germany, 'Zentrum für Humangenetik, MVZ genteQ, Hamburg, Germany, and dDepartment of Genetics and Evolution, Jagiellonian University, Kraków, Poland Key words: array comparative genomic hybridization - copy number variants developmental delay - intellectual disability - microdeletion and microduplication syndromes

Corresponding author: Moneef Shoukier, MD, Institute of Human Genetics, University of Göttingen, Heinrich-Düker-Weg 12,

37073 Göttingen, Germany.

Tel.: +49 55139 7593;

fax: +49 551397567 ;

e-mail: moneef.shoukier@med.unigoettingen.de

Received 1 November 2011, revised and accepted for publication 23 January 2012
Developmental delay/intellectual disability (DD/ID) is a common condition and affects $1-3 \%$ of children (1). In a small subset of these patients, clinical features indicate a monogenic disorder or a numeric or structural change in one or more chromosomes, e.g. a trisomy or an unbalanced translocation. In these patients, clinical genetic 


\section{Shoukier et al.}

testing comprises mutation analyses of single genes or chromosome analysis. For all patients without clinical hallmark pointing to a specific genetic condition, conventional karyotyping or subtelomeric analysis represented the first diagnostic step in the past. During the last few years, array comparative genomic hybridization (array CGH) has revolutionized the diagnostic approach to children with unexplained DD/ID and congenital malformations and has become the first-tier investigation in those patients $(2,3)$.

Array CGH technologies enable a 100-fold higher resolution compared with conventional karyotyping. Recent studies point to an average diagnostic yield of chromosomal microarray analyses of $15-20 \%$ (3). Thus, array CGH shows a much higher sensitivity in detection of chromosomal aberrations than conventional karyotyping, but it still fails to reveal any clinically relevant CNV in the vast majority of patients tested. Array $\mathrm{CGH}$ is expensive and labour-intensive, and clinical criteria allowing for separation of patients with high probability of significant CNV would be desirable. Our study aimed at both enlarging the phenotype spectrum associated with clinically relevant $\mathrm{CNVs}$ and delineating clinical criteria, which may help separating patients with pathogenic $\mathrm{CNV}$ from those without pathogenic CNV.

\section{Patients and methods}

In a retrospective review of data, we included all patients $(n=342)$ who were tested using array CGH as part of evaluation for unexplained DD/ID during a 3.5-year period (September 2007-March 2011) in our outpatients department of paediatric neurology in a tertiary care university hospital. We selected patients for array CGH on the basis of the following criteria: (i) unexplained DD/ID with or without additional features, (ii) parental request to elucidate the aetiology of their child's DD/ID, and (iii) parental willingness to provide own blood samples for array $\mathrm{CGH}$ to determine the inheritance in case a CNV was detected in their child. These criteria were fulfilled by all 342 patients (227 boys and 115 girls).

Total genomic DNA was prepared from ethylenediaminetetraacetic acid (EDTA) blood samples using standard techniques. A genome-wide copy number scan was performed using either the Agilent 244K Arrays or the Agilent SurePrint G3 Human CGH Microarray Kit $4 \times 180 \mathrm{~K}$ (Agilent Technologies, Inc., Santa Clara, CA) according to the manufacturer's instructions. Array data were analysed using the Agilent DNA Analytics 4.0 software. We compared our findings to known CNVs listed in the Database of Genomic Variants (DGV, http://projects.tcag.ca/variation), in the International Standard Cytogenomic Array Consortium Databases (https://isca.genetics.emory.edu) as well as in the Database of Chromosomal Imbalance and Phenotype in Humans using Ensembl Resources (DECIPHER, https://decipher.sanger.ac.uk/application/). Pathogenic or potentially pathogenic CNVs identified by array

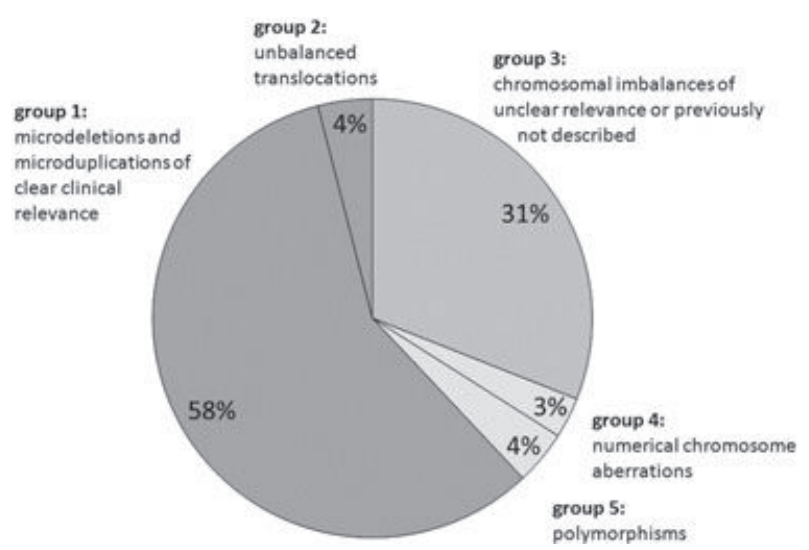

Fig. 1. Allocation of all 72 copy number variants to different groups of genetic aberration.

CGH were verified either by multiplex ligationdependent probe amplification (MLPA, MRC-Holland, Amsterdam, The Netherlands), in case the appropriate kit was available, or alternatively by quantitative real-time polymerase chain reaction (qPCR). Primer sequences and real-time qPCR conditions are available upon request.

Clinical features and body measurements were assessed using a standardized protocol (excel sheet) for collection of data in all children. All statistical analyses were performed with Statistica 9.1 software (StatSoft Inc., Tulsa, OK). Clinical data of children with $\mathrm{CNVs}$ and translocations of clear clinical significance were compared to those with normal array CGH results using the chi-squared test. If the expected number was below 5, the chi-squared test with Yates correction was applied. Bonferroni correction was applied to assess the significance level.

This study received approval by the local institutional review board. Written informed consent was obtained from all families.

\section{Results}

Chromosomal imbalances (CNVs) were found in 72 of 342 children $(21.1 \%$ ) with DD/ID. Of these, 49 were males and 23 were females. The detected CNVs were assigned to one of the following five groups (Fig. 1): group 1: microdeletions and microduplications of clear clinical relevance $(42 / 72=58 \%)$; group 2 : unbalanced translocations $(3 / 72=4 \%)$; group 3: chromosomal imbalances of unclear relevance or previously not described $(22 / 72=31 \%)$; group 4 : numerical chromosome aberrations $(2 / 72=3 \%)$; group 5 : polymorphisms $(3 / 72=4 \%)$. Table 1 summarizes clinical and genetic features of all children with detected CNVs.

Comparison of children with chromosomal microdeletion or microduplication syndromes and translocations of clear clinical relevance (groups 1 and 2,n=45) to those without $(n=270)$ showed that congenital anomalies were statistically more frequent in children with pathogenic CNVs (p-value 0.003). Heart defects were 
Phenotypic clues to clinically relevant CNVs

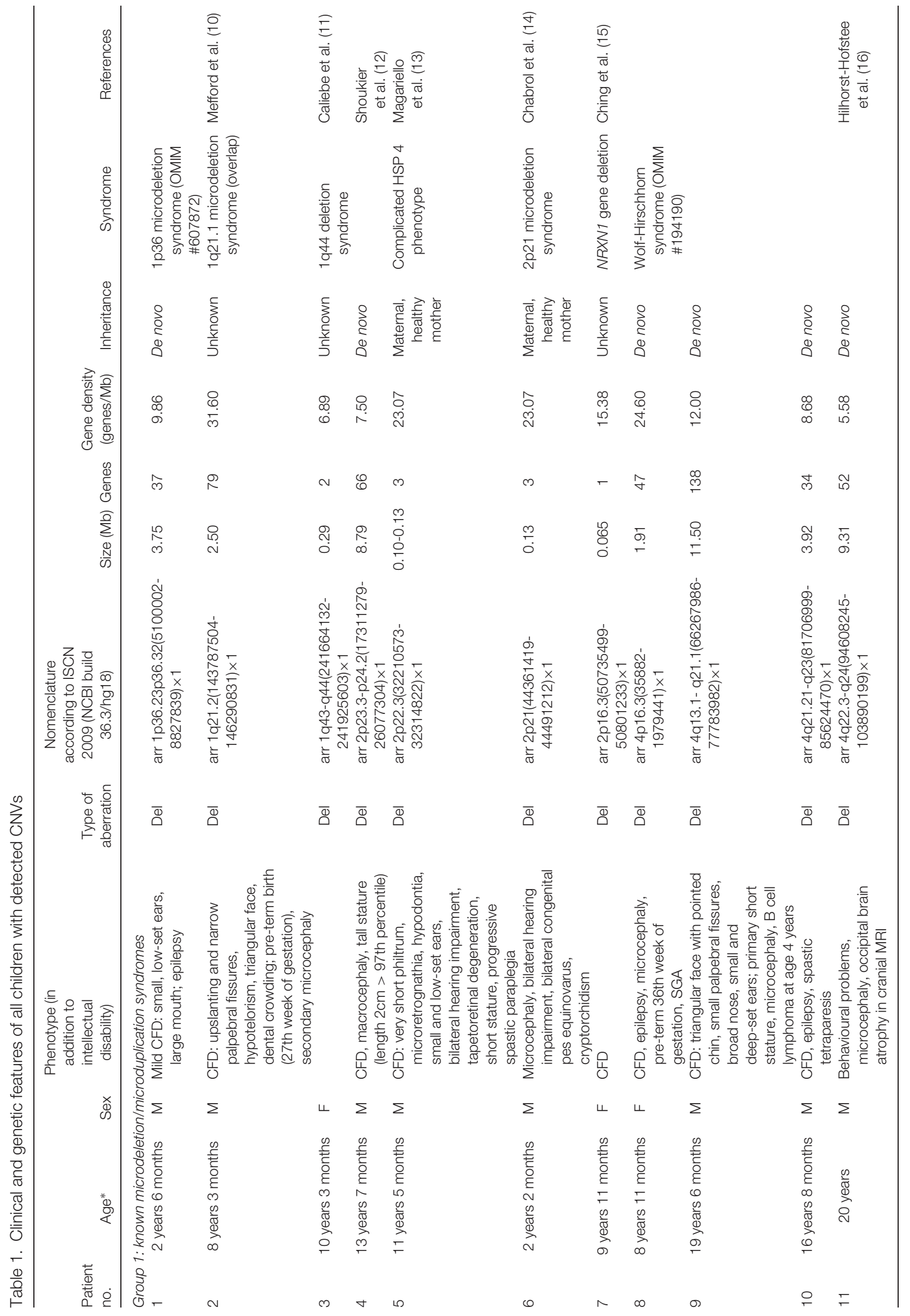


Shoukier et al.

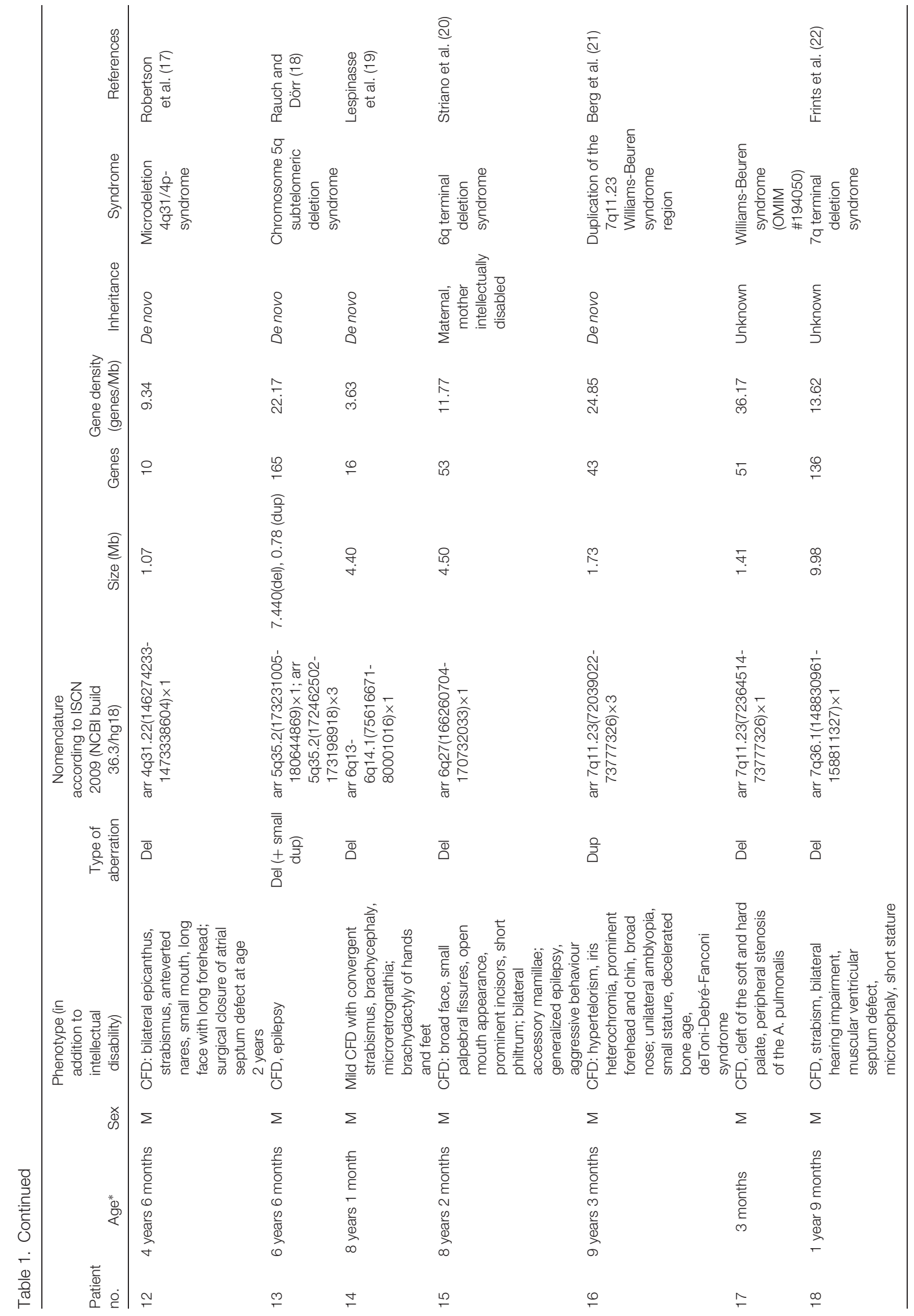


Phenotypic clues to clinically relevant CNVs

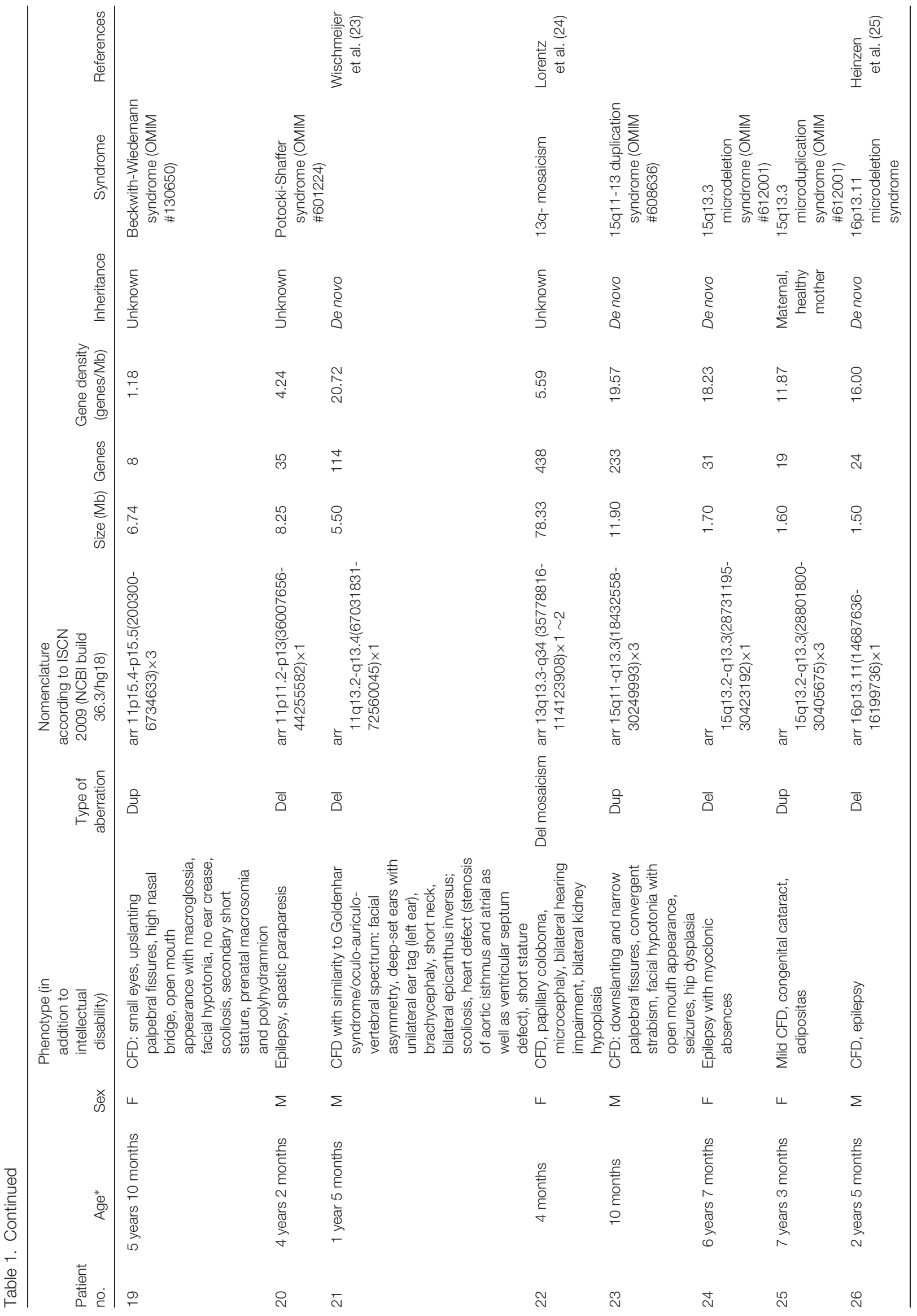


Shoukier et al.

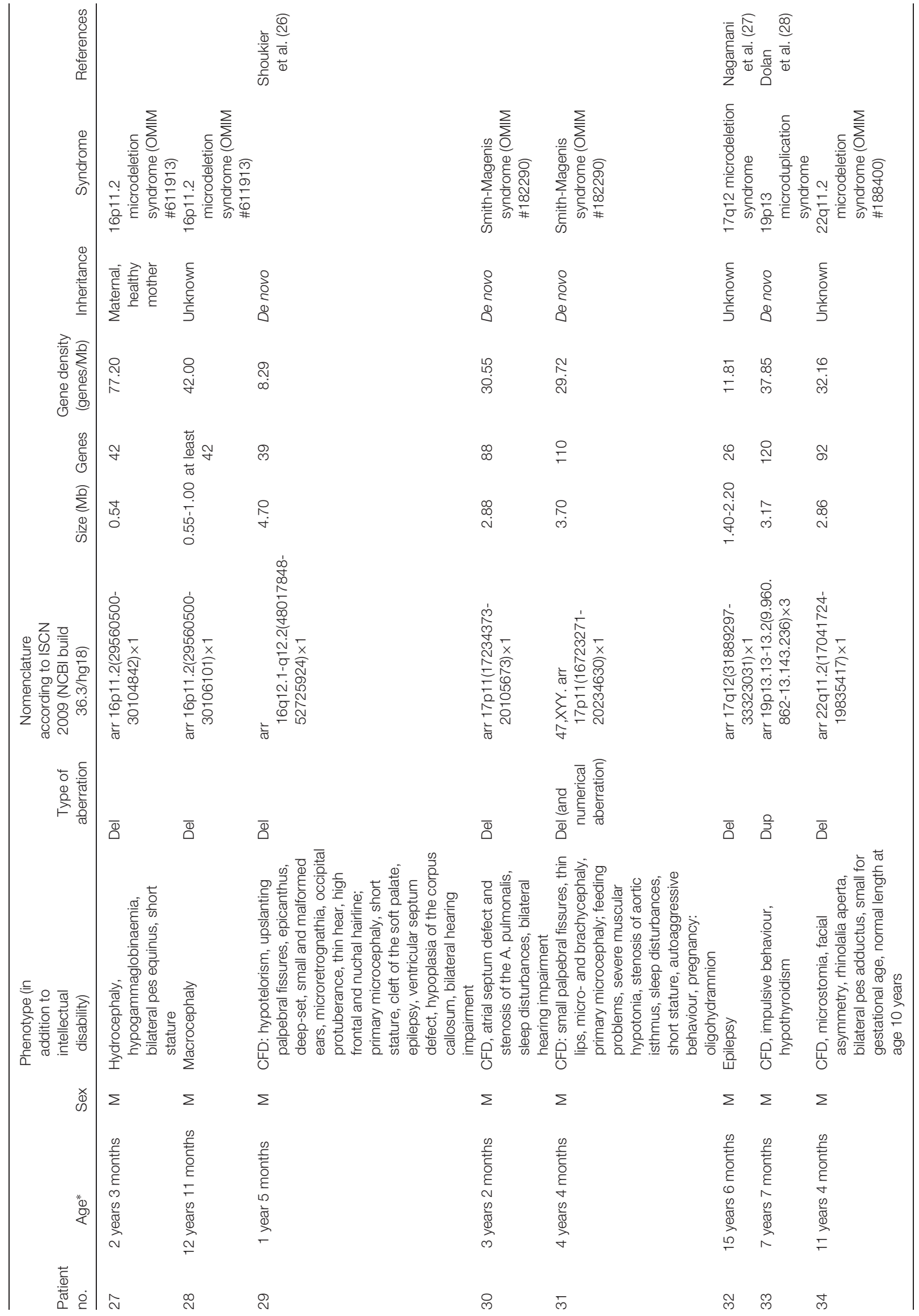


Phenotypic clues to clinically relevant CNVs

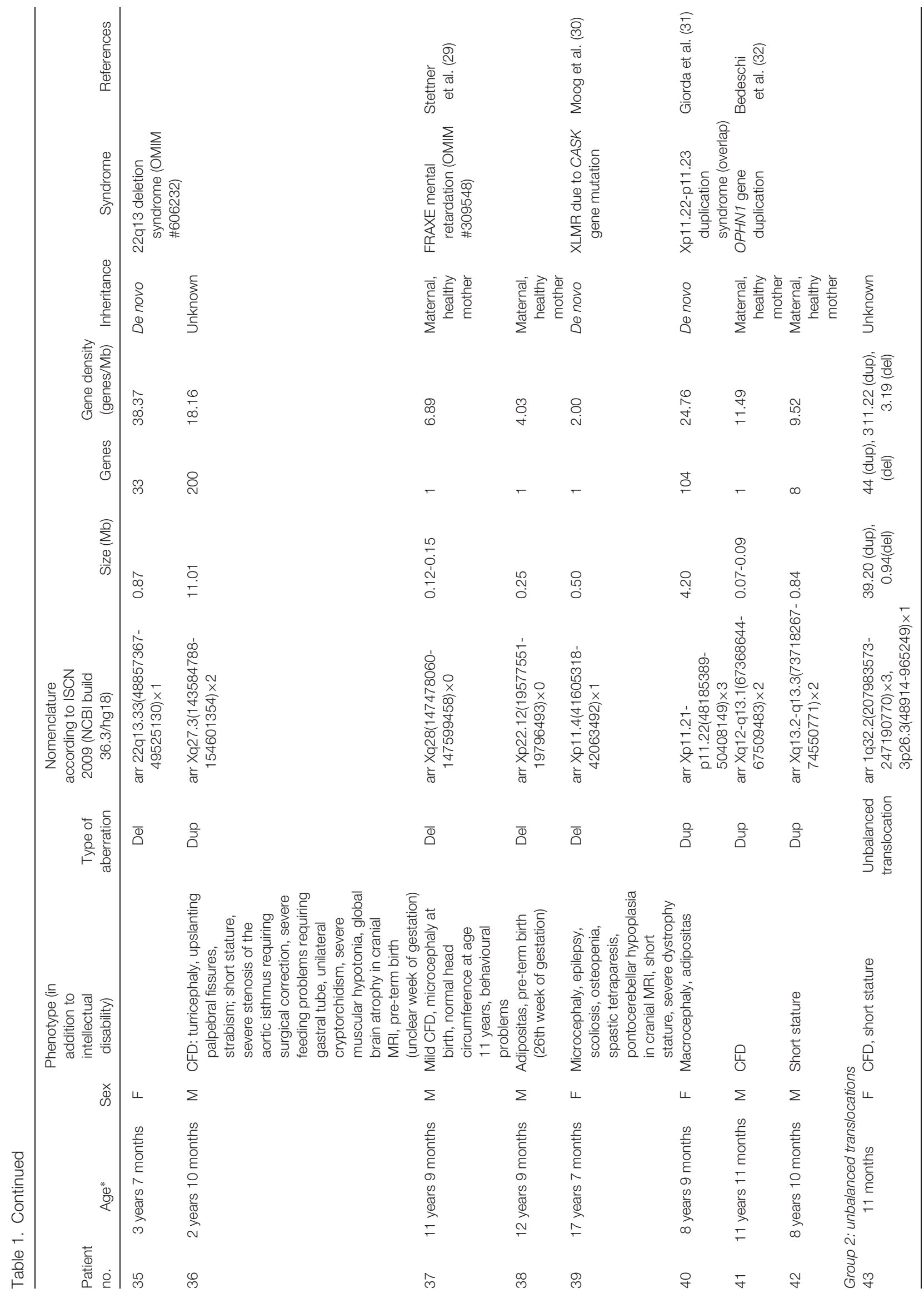


Shoukier et al.

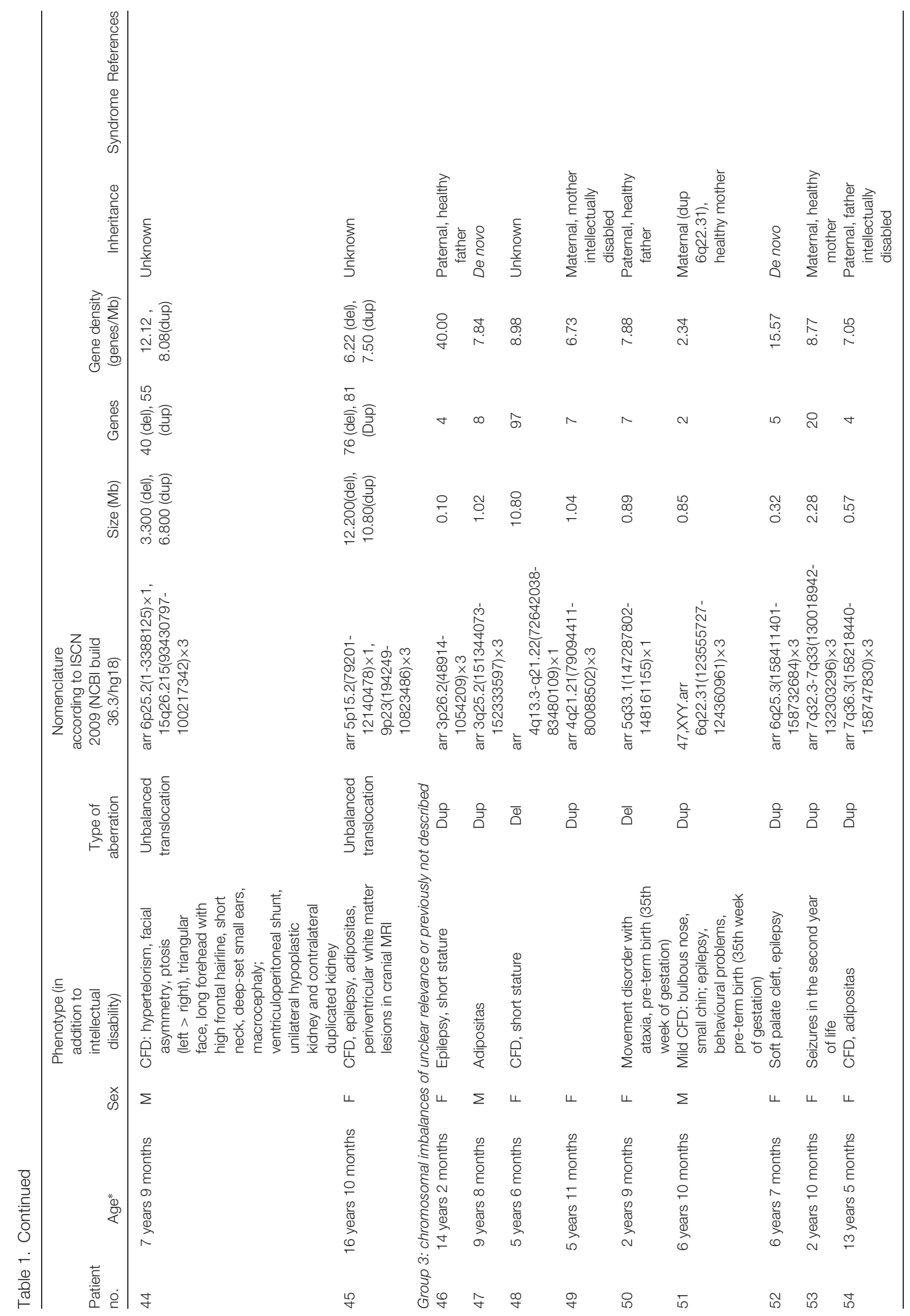


Phenotypic clues to clinically relevant CNVs

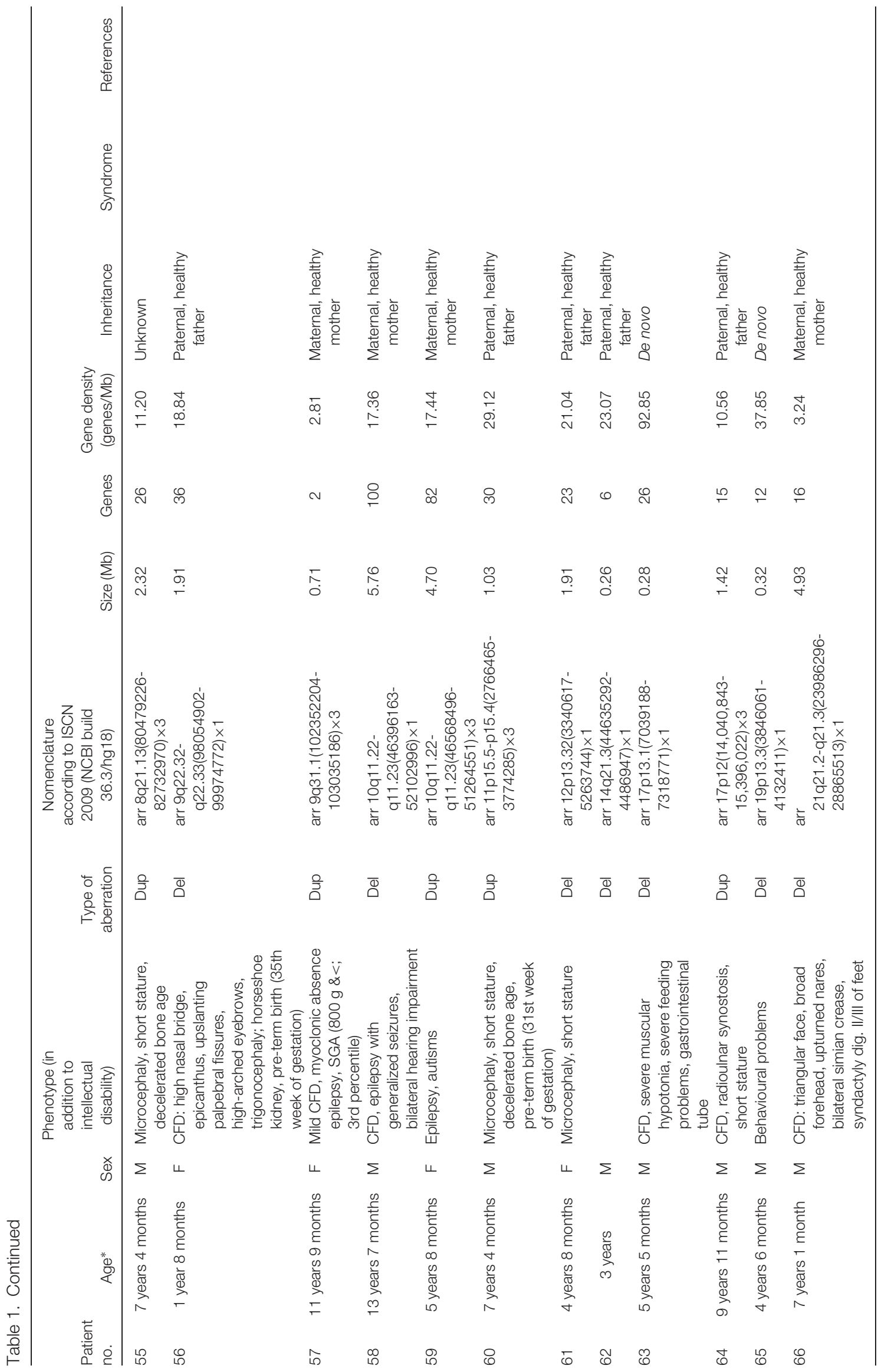


Shoukier et al.

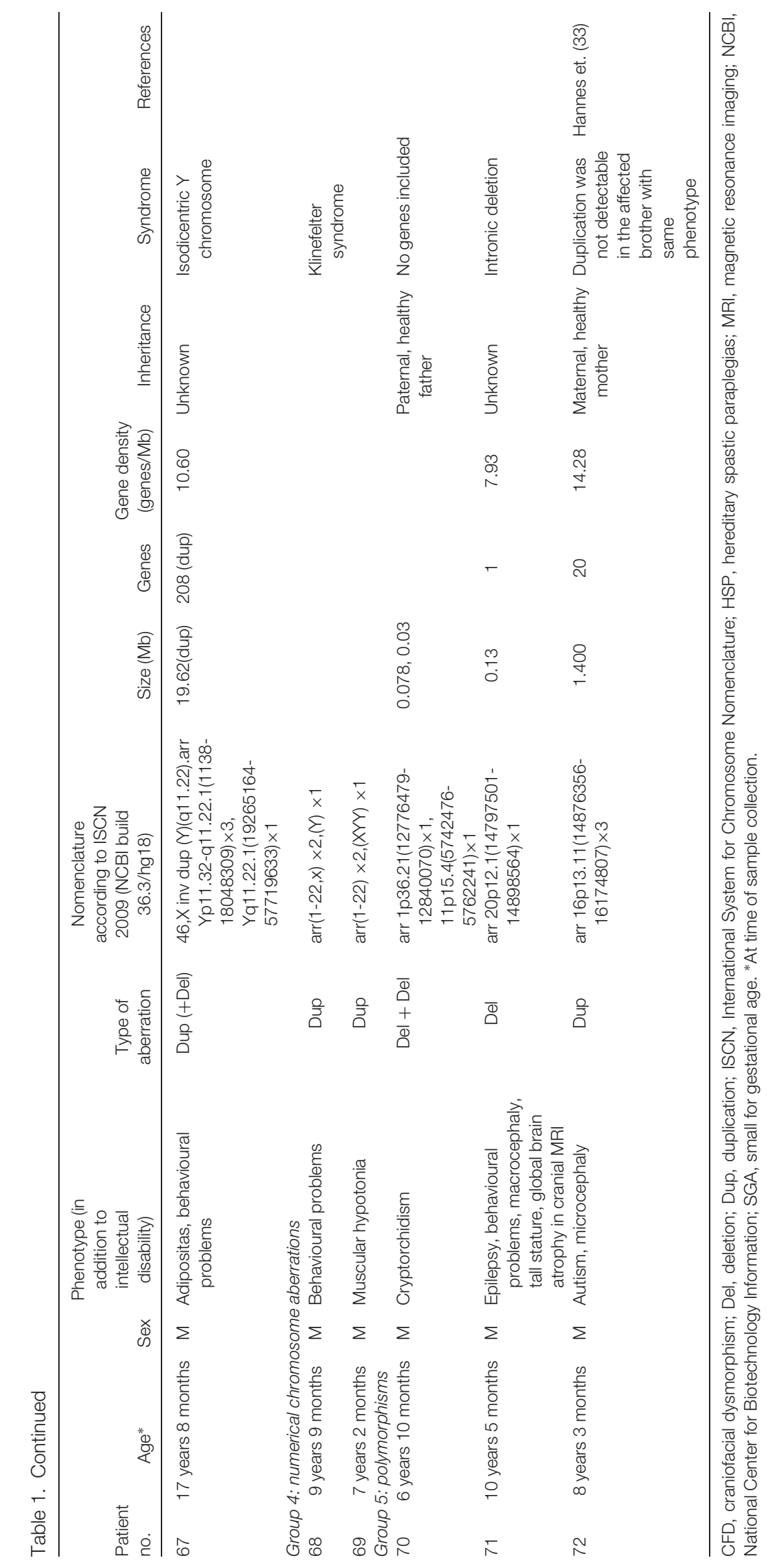



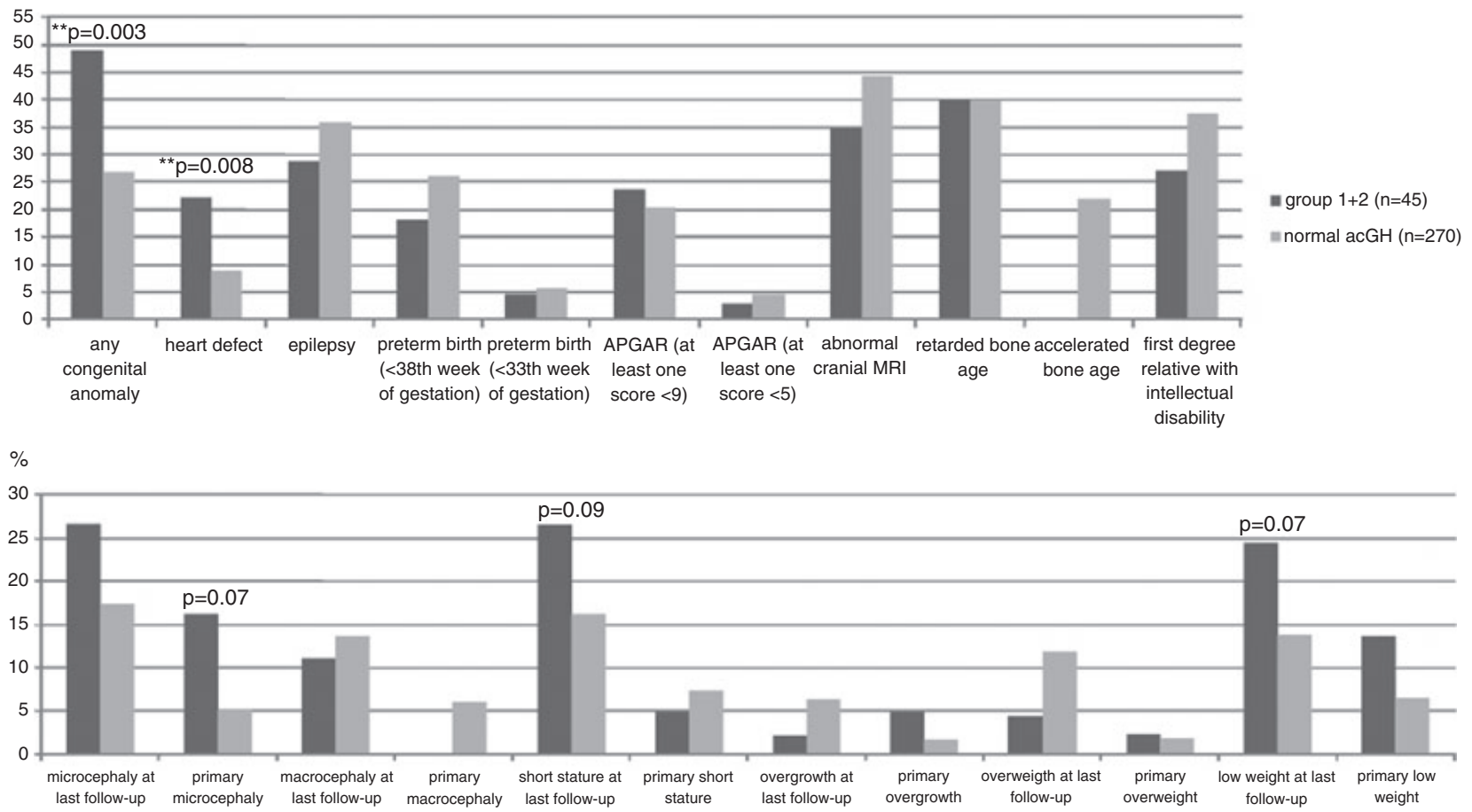

Fig. 2. Evaluation of clinical data in children with copy number variant $(\mathrm{CNV})$ of clear clinical significance (groups $1+2)$ in comparison with children with normal array comparative genomic hybridization (array CGH) analysis. Bold p-values with double asterisk (**) indicate statistically significant differences.

the most frequent congenital anomaly and were significantly more frequent in the group with clinically relevant CNVs (p-value 0.008). With respect to body measurements at birth as well as at most recent followup, primary microcephaly (p-value 0.07 ), short stature at most recent follow-up (p-value 0.09) as well as low weight at most recent follow-up (p-value 0.07) were more frequent in children with chromosomal aberrations, but these differences did not reach statistical significance. Of note, epilepsies, macrocephaly and overgrowth were more frequent in children with normal array CGH results, however, again without statistical significance (Fig. 2). Figure 3 displays facial phenotypes of several patients with CNVs of certain and uncertain clinical significance detected by array CGH.

Inheritance of structural aberrations (groups 1-3) was analysed in $72 \%(48 / 67)$ of patients. In 25 children, the chromosomal imbalance was not found in the parents and therefore occurred de novo, whereas 23 children inherited their structural chromosomal imbalance from one parent. Most parents carrying the same chromosomal imbalance as their affected child were healthy, whereas three parents were intellectually disabled (parents of patients 15, 49 and 54).

\section{Discussion}

In our study, array CGH in 342 children with $\mathrm{DD} / \mathrm{ID}$ revealed chromosomal imbalances (CNVs) in 72 patients $(21.1 \%)$. Of these, microdeletions and microduplications as well as unbalanced translocations were found in approximately two thirds (45/72), thus enabling a clear aetiological diagnosis in $13.2 \%$ of all analysed children (45/342, groups $1+2$, Fig. 1 , Table 1). However, $6.4 \%$ of all children (22/342, group 3 ) had chromosomal imbalances of yet unclear clinical relevance. Our diagnostic yield of pathogenic CNVs is comparable to the yields obtained from two large studies that used similar array platforms $(14.2 \%$ and $14.7 \%$; (4) and (5), respectively). In addition to array resolution, patient selection seems to have the largest influence on the diagnostic yield of array $\mathrm{CGH}$. Our cohort comprised a large proportion of children who underwent previous diagnostic procedures that already excluded a wide range of other causes of DD/ID. All children had routine blood tests and metabolic screening tests, such as amino acids in plasma and organic acids in urine. Analysis of cerebrospinal fluid was performed in $80 \%$ of children, and $70 \%$ had conventional karyotyping and subtelomere screening before array $\mathrm{CGH}$ analysis.

Statistical evaluation of clinical data revealed that congenital anomalies, especially heart defects, were significantly more frequent in children with chromosomal microdeletion or microduplication syndromes and translocations of clear clinical relevance (groups $1+2$ ) compared to children with normal array CGH results (Fig. 2). Further clinical criteria indicating a higher probability of a chromosomal aberration causative for DD/ID were primary microcephaly, short stature and low weight at most recent follow-up, although statistical significance was not reached. This higher frequency 


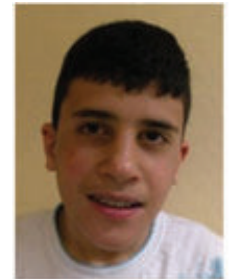

patient 1

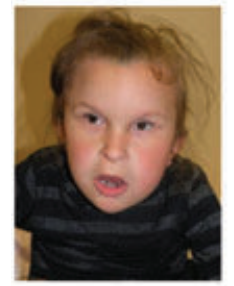

patient 19

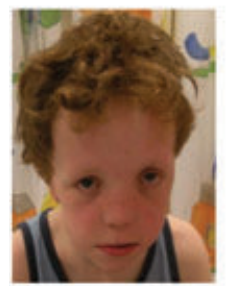

patient 44

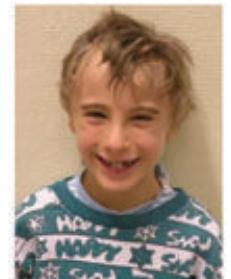

patient 2

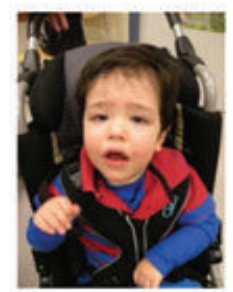

patient 23

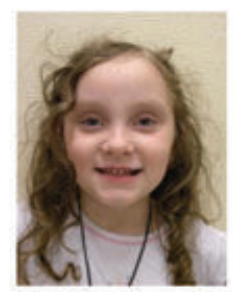

patient 49

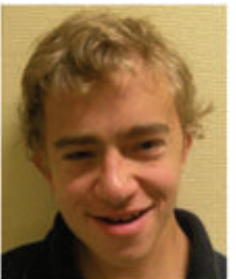

patient 9

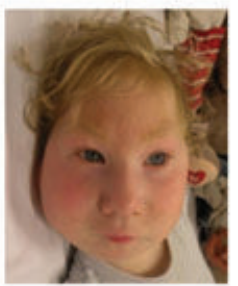

patient 29

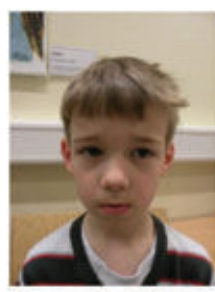

patient 51

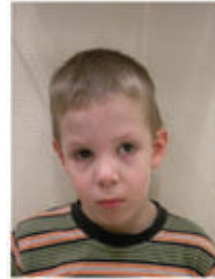

patient 12

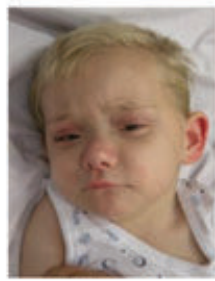

patient 31

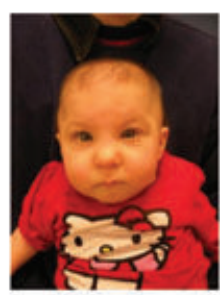

patient $56(7 \mathrm{~m})$

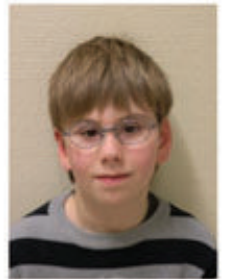

patient 14

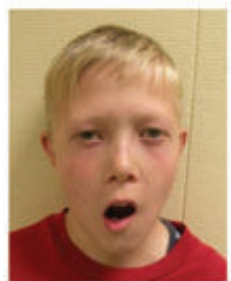

patient 34

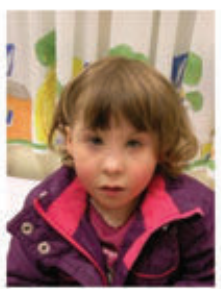

patient $56(3 y)$

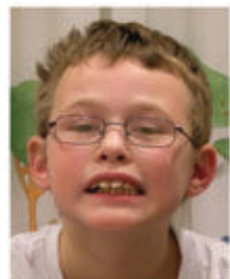

patient 15

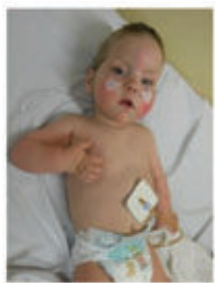

patient 36

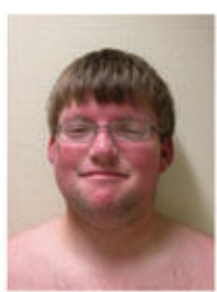

patient 67

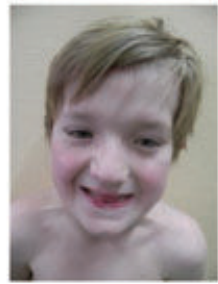

patient 16

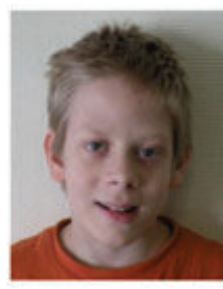

patient 42

Fig. 3. Facial phenotypes of children with copy number variants (CNVs) detected by array comparative genomic hybridization (array CGH). For clinical and genetic details, see Table 1. Parental/guardian informed consent was obtained for publication of these photos.

of pathogenic CNVs in individuals with congenital anomalies has also been observed in previous studies $(4,6,7)$. However, the phenotypic spectrum in chromosomal aberrations is very broad, and causative chromosomal aberrations were also found in children with pure DD/ID. Therefore, array CGH should not be restricted to children with $\mathrm{DD} / \mathrm{ID}$ and additional congenital anomalies, but seems to be an important diagnostic tool in every child with DD/ID independent of additional features. The same loosening of recommended selection criteria was previously experienced with the de Vries criteria in subtelomere fluorescence in situ hybridization (FISH) studies (8).

Summarizing the results in our cohort, approximately two thirds of the detected CNVs correspond to previously known microdeletion or microduplication syndromes or chromosomal translocations and therefore permit a clear aetiological diagnosis of DD/ID in the affected children (groups $1+2$ ). The clinical relevance of $31 \%$ of detected CNVs (group 3) remains uncertain at present because of the following reasons: (i) the $\mathrm{CNV}$ is previously unknown and has neither been found and published in affected nor in healthy controls and (ii) the CNV is inherited from a healthy parent, which hampers its classification as pathogenic variant with incomplete penetrance or familial polymorphism. However, several of these chromosome imbalances with yet unclear clinical significance may represent emerging recurrent chromosomal microaberration syndromes.
Thus, it is important to collect and publish further data on CNVs with the corresponding detailed clinical phenotype. Overlapping imbalances can result in new syndromes by the 'reverse phenotypics' approach, in which a similar genomic aberration precedes the definition of a similar clinical presentation (9).

\section{Acknowledgements}

We thank S. Görlich, J. Kahlert and S. Vogel for their help with clinical data collection. We thank S. Herold and M. Steckel for excellent technical assistance.

\section{References}

1. Roeleveld N, Zielhuis GA, Gabreels F. The prevalence of mental retardation: a critical review of recent literature. Dev Med Child Neurol 1997: 39: 125-132.

2. Manning M, Hudgins L. Array-based technology and recommendations for utilization in medical genetics practice for detection of chromosomal abnormalities. Genet Med 2010: 12: 742-745.

3. Miller DT, Adam MP, Aradhya S et al. Consensus statement: chromosomal microarray is a first-tier clinical diagnostic test for individuals with developmental disabilities or congenital anomalies. Am J Hum Genet 2010: 86: 749-764.

4. Cooper GM, Coe BP, Girirajan S et al. A copy number variation morbidity map of developmental delay. Nat Genet 2011: 43: 838-846.

5. Kaminsky EB, Kaul V, Paschall J et al. An evidence-based approach to establish the functional and clinical significance of copy number variants in intellectual and developmental disabilities. Genet Med 2011: 13: $777-784$. 


\section{Phenotypic clues to clinically relevant CNVs}

6. Baris HN, Tan WH, Kimonis VE, Irons MB. Diagnostic utility of array-based comparative genomic hybridization in a clinical setting. Am J Med Genet A 2007: 143A: 2523-2533.

7. Lu XY, Phung MT, Shaw CA et al. Genomic imbalances in neonates with birth defects: high detection rates by using chromosomal microarray analysis. Pediatrics 2008: 122: 1310-1318.

8. de Vries BB, White SM, Knight SJ et al. Clinical studies on submicroscopic subtelomeric rearrangements: a checklist. J Med Genet 2001: 38: $145-150$.

9. Vissers LE, de Vries BB, Veltman JA. Genomic microarrays in mental retardation: from copy number variation to gene, from research to diagnosis. J Med Genet 2010: 47: 289-297.

10. Mefford HC, Sharp AJ, Baker C et al. Recurrent rearrangements of chromosome 1q21.1 and variable pediatric phenotypes. N Engl J Med 2008: 359: 1685-1699.

11. Caliebe A, Kroes HY, van der Smagt JJ et al. Four patients with speech delay, seizures and variable corpus callosum thickness sharing a 0.440 $\mathrm{Mb}$ deletion in region 1q44 containing the HNRPU gene. Eur J Med Genet 2010: 53: 179-185.

12. Shoukier M, Schroder J, Zoll B et al. A de novo interstitial deletion of 2p23.3-24.3 in a boy presenting with intellectual disability, overgrowth, dysmorphic features, skeletal myopathy, dilated cardiomyopathy. Am J Med Genet A 2012: 158: 429-433.

13. Magariello A, Muglia M, Patitucci A et al. Mutation analysis of the SPG4 gene in Italian patients with pure and complicated forms of spastic paraplegia. J Neurol Sci 2010: 288: 96-100.

14. Chabrol B, Martens K, Meulemans S et al. Deletion of C2orf34, PREPL and SLC3A1 causes atypical hypotonia-cystinuria syndrome. J Med Genet 2008: 45: 314-318.

15. Ching MS, Shen Y, Tan WH et al. Deletions of NRXN1 (neurexin-1) predispose to a wide spectrum of developmental disorders. Am J Med Genet B Neuropsychiatr Genet 2010: 153B: 937-947.

16. Hilhorst-Hofstee $\mathrm{Y}$, Tumer Z, Born $\mathrm{P}$ et al. Molecular characterization of two patients with de novo interstitial deletions in 4q22-q24. Am J Med Genet A 2009: 149A: 1830-1833.

17. Robertson SP, O'Day K, Bankier A. The 4q-syndrome: delineation of the minimal critical region to within band 4q31. Clin Genet 1998: 53: 70-73.

18. Rauch A, Dorr HG. Chromosome 5 q subtelomeric deletion syndrome. Am J Med Genet C Semin Med Genet 2007: 145C: 372-376.

19. Lespinasse J, Gimelli S, Bena F, Antonarakis SE, Ansermet F, PaoloniGiacobino A. Characterization of an interstitial deletion 6q13-q14.1 in a female with mild mental retardation, language delay and minor dysmorphisms. Eur J Med Genet 2009: 52: 49-52.

20. Striano P, Malacarne M, Cavani S et al. Clinical phenotype and molecular characterization of $6 \mathrm{q}$ terminal deletion syndrome: five new cases. Am J Med Genet A 2006: 140: 1944-1949.
21. Berg JS, Brunetti-Pierri N, Peters SU et al. Speech delay and autism spectrum behaviors are frequently associated with duplication of the 7q11.23 Williams-Beuren syndrome region. Genet Med 2007: 9: 427-441.

22. Frints SG, Schrander-Stumpel CT, Schoenmakers EF et al. Strong variable clinical presentation in 3 patients with $7 \mathrm{q}$ terminal deletion. Genet Couns 1998: 9: 5-14.

23. Wischmeijer A, Magini P, Giorda R et al. Olfactory receptor-related duplicons mediate a microdeletion at $11 \mathrm{q} 13.2 \mathrm{q} 13.4$ associated with a syndromic phenotype. Mol Syndromol 2011: 1: 176-184.

24. Lorentz CP, Jalal SM, Thompson DM, Babovic-Vuksanovic D. Mosaic $\mathrm{r}(13)$ resulting in large deletion of chromosome $13 \mathrm{q}$ in a newborn female with multiple congenital anomalies. Am J Med Genet 2002: 111: $61-67$.

25. Heinzen EL, Radtke RA, Urban TJ et al. Rare deletions at 16p13.11 predispose to a diverse spectrum of sporadic epilepsy syndromes. Am J Hum Genet 2010: 86: 707-718.

26. Shoukier M, Wickert J, Schroder J et al. A $16 \mathrm{q} 12$ microdeletion in a boy with severe psychomotor delay, craniofacial dysmorphism, brain and limb malformations, and a heart defect. Am J Med Genet A 2011. DOI: 10.1002/ajmg.a.34387.

27. Nagamani SC, Erez A, Shen J et al. Clinical spectrum associated with recurrent genomic rearrangements in chromosome 17q12. Eur J Hum Genet 2010: 18: 278-284.

28. Dolan M, Mendelsohn NJ, Pierpont ME, Schimmenti LA, Berry SA, Hirsch B. A novel microdeletion/microduplication syndrome of 19p13.13. Genet Med 2010: 12: 503-511.

29. Stettner GM, Shoukier M, Hoger C, Brockmann K, Auber B. Familial intellectual disability and autistic behavior caused by a small FMR2 gene deletion. Am J Med Genet A 2011: 155: 2003-2007.

30. Moog U, Kutsche K, Kortum F et al. Phenotypic spectrum associated with CASK loss-of-function mutations. J Med Genet 2011: 48: $741-751$.

31. Giorda R, Bonaglia MC, Beri S et al. Complex segmental duplications mediate a recurrent $\operatorname{dup}(\mathrm{X})(\mathrm{p} 11.22-\mathrm{p} 11.23)$ associated with mental retardation, speech delay, and EEG anomalies in males and females. Am J Hum Genet 2009: 85: 394-400.

32. Bedeschi MF, Novelli A, Bernardini L et al. Association of syndromic mental retardation with an $\mathrm{Xq12q13.1}$ duplication encompassing the oligophrenin 1 gene. Am J Med Genet A 2008: 146A: 1718-1724.

33. Hannes FD, Sharp AJ, Mefford HC et al. Recurrent reciprocal deletions and duplications of 16p13.11: the deletion is a risk factor for MR/MCA while the duplication may be a rare benign variant. J Med Genet 2009: 46: 223-232. 